Revista Brasileira de Informática na Educação - RBIE Brazilian Journal of Computers in Education (ISSN online: 2317-6121; print: 1414-5685) http://br-ie.org/pub/index.php/rbie

Submission: 20/Feb/2021; $\quad 1^{\text {st }}$ round notif.: 05/May/2021; $\quad$ New version: 25/May/2021; $\quad 2^{\text {nd }}$ round notif.: 30/Jun/2021;

Camera ready: 15/Jul/2021; $\quad$ Edition review: 21/Jul/2021;

Available online: 06/Aug/2021;

Published: 06/Aug/2021;

\title{
Desarrollo de la apreciación musical en niños Sordos mediante estimulación por vibraciones desde una aplicación móvil
}

\author{
Title: Development of music appreciation in deaf children through vibration stimulation from \\ a mobile app
}

\author{
Andrés Eduardo Fuentes Cortázar \\ Universidad Veracruzana \\ afuentescortazar@gmail.com
}

\author{
José Rafael Rojano-Cáceres \\ Universidad Veracruzana \\ rrojano@uv.mx
}

\section{Resumen}

La música como un fenómeno acústico, se encuentra relacionada estrechamente con el sentido del oído, motivo por el cual posiblemente resulte difícil concebir que las personas con discapacidad (PCD) auditiva profunda o personas sordas puedan ser capaces de comprender los conceptos asociados a dicho fenómeno. Para una persona que vive sin discapacidad auditiva el acceder a los componentes musicales y desarrollarse en muchos casos en dicha área resulta natural. No obstante, la enseñanza de la música y sus conceptos por métodos tradicionales no resulta factible para el caso de PCD auditiva. Para ese caso en particular será mejor recurrir a otros sentidos, como puede ser el caso del sentido del tacto. Por lo tanto, en el presente artículo de investigación se describe el proceso a través del cual se aborda la enseñanza de conceptos asociados a la música en un grupo de niños sordos a través de una aplicación móvil, empleando la estimulación a través de las vibraciones generadas por los teléfonos móviles. Sin embargo, debido a las condiciones imperantes de la pandemia causada por COVID-19, la investigación debió llevarse de forma remota teniendo la posibilidad de monitorear solo a una muestra de los niños del grupo original, mediante el seguimiento de las interacciones con la aplicación.

Palabras-Clave: Inclusión; Sordo; Música; Vibrotáctil; Aplicación Móvil

\begin{abstract}
Music as an acoustic phenomenon is closely related to the sense of hearing, which is why it is possibly difficult to conceive that people with profound hearing disabilities or deaf people may be able to understand the concepts associated with this phenomenon. For a person who lives without hearing impairment, accessing the musical components and developing in this area in many cases is natural. However, the teaching of music and its concepts by traditional methods is not feasible in the case of people with auditory disability. For that particular case, it will be better to resort to other senses, such as the sense of touch. Therefore, this research article describes the process through which the teaching of concepts associated with music in a group of deaf children is approached through a mobile application, using stimulation through the vibrations generated by mobile phones. Nevertheless, due to the prevailing conditions of the pandemic caused by COVID-19, the investigation had to be carried out remotely, having the possibility of monitoring only a sample of the children of the original group, by monitoring interactions with the application.
\end{abstract}

Keywords: Inclusion; Deaf; Music; Vibro-Tactile; Mobile App

Cite as: Fuentes Cortázar, A. E., \& Rojano-Cáceres, J. R. (2021). Desarrollo de la apreciación musical en niños Sordos mediante estimulación por vibraciones desde una aplicación móvil. Revista Brasileira de Informática na Educação, 29, 1007-1037. DOI: 10.5753/RBIE.2021.29.0.1007. 


\section{Introducción}

Cuando se piensa en el concepto de música, por lo menos en la cotidianidad, se le asocia de forma inmediata con el sentido de la audición. Formalmente, se define a ésta como el arte de fusionar sonidos en una secuencia temporal definida, contemplando las leyes de la armonía, la melodía y el ritmo, o de producirlos mediante instrumentos musicales y que por lo habitual reproducen un efecto agradable y expresivo (Oxford, 2019). Quizás por esta definición puede resultar difícil de asociar con personas que carezcan de dicho sentido como es el caso de las personas que viven con una discapacidad auditiva o personas sordas. Esta forma de pensamiento puede generar la exclusión en actividades relacionadas con la música para dichas personas.

Fourney y Fels, bosquejan el hecho de que la música no es ajena a las personas sordas, pero sí representa una experiencia limitada, destacando que la experiencia le puede ser transmitida a ellos a través de estímulos visuales (Fourney y Fels, 2009). En la experiencia empírica de los autores, también consta el hecho de que la comunidad sorda desarrolla su propia interpretación de las letras de las canciones haciendo uso de la lengua de señas.

De acuerdo con la confederación Fomentando Inclusión Apoyando Personas Avanzando Solidariamente (FIAPAS) de España, la sordera se define como aquella pérdida o anomalía de una función fisiológica o anatómica del sistema auditivo, causando una discapacidad o dificultad para poder escuchar. En otros términos, se dice que una persona es sorda cuando ésta posee un problema en uno o ambos órganos auditivos, provocando un impedimento total o parcial auditivo (FIAPAS, 2005).

Por su parte el Consejo Nacional para el Desarrollo y la Inclusión de las Personas con Discapacidad (CONADIS) de México, comenta que cuando una persona nace o adquiere una discapacidad auditiva ésta se ve limitada en términos de comunicación con las personas oyentes que le rodean y como consecuencia, la persona es restringida a no desarrollar una vida social digna e inclusiva (CONADIS, 2016).

Otro rasgo particular de la comunidad de personas con discapacidad auditiva, es el hecho de que para comunicarse entre ellos emplean una comunicación del tipo viso-gestual, a través de su lengua propia denominada Lengua de Señas (LS). Por las características propias de dicha lengua al no ser transmitida oralmente no siempre facilita la posibilidad de relacionarse con las personas oyentes, que por regla desconocen la lengua.

Por ello, el presente trabajo de investigación busca sentar las bases para que niños con discapacidad auditiva profunda logren acceder a los conceptos relacionados con la música desde una perspectiva donde no se dependa del sentido del oído, sino del sentido del tacto. De esta forma, se describe la investigación realizada inicialmente de forma presencial con un grupo de 12 niñas y niños sordos con edades variables entre 5 y 12 años, en donde se sentaron los conceptos necesarios para posteriormente poder reforzarlos a través del uso de una aplicación móvil. Cabe señalar que la fase de interacción con la aplicación móvil se vio interrumpida por la repentina amenaza de la contingencia sanitaria COVID-19, debido a ello el grupo de usuarios se limitó a 5 niños a quienes finalmente se les da seguimiento remoto de sus interacciones con la aplicación. Finalmente, cabe decir que en este trabajo nos referimos a las personas con discapacidad auditiva también como personas sordas, ya que no representan una forma de discriminación en el uso del lenguaje para hacer referencia a ellos o a su comunidad. 


\section{Antecedentes}

A lo largo del tiempo, diversos investigadores han intentado dar una explicación al respecto sobre si una persona sorda pudiera percibir la música o no. Aunado a ello, en el artículo de Liliana Otero llamado "La sordera: una oportunidad para describir la música", pretende sostener que la música va más allá de sólo percibirla con el oído. El sonido, además de ser un fenómeno acústico que llega al sistema auditivo, es reconocido por la producción de la vibración originada por un cuerpo y difundida mediante ondas (Caicedo, 2015).

Fisiológicamente, el oído humano logra percibir estos sonidos a través de una gama de frecuencias que comprende desde los 15 hasta $20.000 \mathrm{~Hz}$. A los sonidos que están debajo de los $15 \mathrm{~Hz}$ se le llaman infrasonidos, mientras que a los sonidos que están encima de los $20.000 \mathrm{~Hz}$ se les llaman ultrasonidos. En el caso de la música, los sonidos más agudos suelen llegar a los $5.000 \mathrm{~Hz}$ (Tomasini, 2007). Para determinar si una persona tiene algún grado de pérdida auditiva se le debe practicar un examen especial basado en un audiograma. En este examen los rangos de prueba van de $250 \mathrm{~Hz}$ a $8000 \mathrm{~Hz}$ que es generalmente la frecuencia del habla humana. Para una persona que tiene una pérdida auditiva de moderada a profunda, el nivel de volumen que no es capaz de percibir está entre los 41 hasta los $91 \mathrm{Db}$ (Cuena, 2015). Por otro lado, las personas que se comunican empleando la lengua de señas probablemente no son capaces de escuchar sonidos más bajos de $95 \mathrm{Db}$ (Hash, 2003).

De esta forma, el sonido presenta cuatro cualidades particulares: el timbre, el cual permite diferenciar sonidos de voces y diversos instrumentos musicales; la intensidad, que muestra la magnitud o fuerza del sonido pudiendo ser fuerte o débil; la duración, relacionada con el tiempo que establece los ritmos musicales; y por último, la altura, la cual permite la diferenciación de sonidos graves con agudos. Por otra parte, la música presenta elementos estructurales característicos: el ritmo, siendo una combinación de sonidos conforme a sus respectivas duraciones; la armonía, la cual coordina los sonidos de forma simultánea; y la melodía, siendo la mezcla de sonidos de acuerdo con las notas de un ritmo determinado (Caicedo, 2015).

De acuerdo con lo anterior, la música no requiere únicamente de la audición para lograr ser percibida, ya que existe una relación entre ambos conceptos anteriormente mencionados, teniendo como base fundamental a la vibración. Por ello, no es equívoco especular que una persona con discapacidad auditiva logre percibir y apreciar la música mediante su cuerpo, dado que el sonido es una vibración que puede ser percibida mediante la piel, y particularmente, se puede percibir a través del sentido del tacto.

\subsection{Trabajos relacionados}

La enseñanza de la música para personas con discapacidad no es nueva, en particular para personas con discapacidad auditiva se pueden encontrar registros que datan desde poco más de 170 años (Darrow, 1985). La enseñanza de la música a personas con discapacidad (PCD) requiere considerar ajustes, adecuaciones o estrategias, como pueden ser enfocarse en que los niños sientan las vibraciones de instrumentos como el piano, el tambor o el bajo. También se pueden transmitir las vibraciones a través de la colocación de un globo situado frente a una bocina, así como la percepción de las bajas frecuencias de instrumentos musicales al sostenerlos con las manos. Generalmente uno de los métodos más socorridos consiste en el uso de percusiones que proveen una 
retroalimentación auditiva más larga (McDowell, 2010). Diversos trabajos que consideran la enseñanza de la música en niños sordos tienen como objetivo el reforzar la adquisición del lenguaje, entre estos se puede consultar (Hash, 2003), (Darrow, 2007), o (Torppa y Huotilainen, 2019); entre los estudios consultados para conocer la relación que hay entre música y discapacidad auditiva se puede citar a (Innes-Brown, Marozeau, Storey, y Blamey, 2013) quien realizó un estudio con niños con diversos grados de discapacidad auditiva, quienes también tenían auxiliares auditivos o implantes cocleares. En dicho estudio se encontró como conclusión más relevante que el desempeño de niños con discapacidad auditiva puede ser tan bueno como en aquellos que no tienen discapacidad en las actividades musicales que implican un fuerte componente rítmico.

En relación con el uso de la tecnología también se ha tomado a ésta en consideración como un factor importante para el apoyo a niños con alguna discapacidad. De forma general, sin tocar el tema de la discapacidad auditiva, McCord describe una gama de programas para computadora que permiten auxiliar en la enseñanza de la música (McCord, 2001).

Dentro de los esfuerzos en la enseñanza de la música a PCD auditiva se puede señalar el trabajo de Music and the Deaf que es una organización benéfica fundada en 1998 en el Reino Unido. Dicha organización es dirigida por Danny Lane, siendo él un pianista con discapacidad auditiva, quien en sus propias palabras ha logrado solidificar una reputación internacional por superar los límites de lo que las personas con sordera pueden aspirar y lograr en la música (Music and the Deaf, 2019).

En el caso de los autores Karam, Russo y Fels, trabajaron en la elaboración de un modelo de cóclea humana que es utilizado para traducir música en señales de vibración, y esto lográndose con una retroalimentación visual a través de la ayuda de una pantalla. El principal objetivo del trabajo de los autores anteriormente mencionados es fomentar la inclusión y mejorar la accesibilidad a la información emocional expresada en música para personas que viven con una discapacidad auditiva (Karam, Russo, y Fels, 2009).

Asimismo, el proyecto Musical Vibrations, de la Unidad de Investigación de Acústica de la Universidad de Liverpool, tiene como propósito llevar la música a personas que viven con discapacidad auditiva, explotando las cualidades y alcances de la vibración. El proyecto trabaja mediante una metodología de enseñanza para personas con discapacidad auditiva y artefactos peculiares que funcionan como receptores para transmitir la vibración de diversos instrumentos musicales (Musical Vibrations, 2010).

Para el caso de niños que cuentan con auxiliares auditivos, o específicamente, implantes cocleares (IC) (Zhou, Sim, Tan, y Wang, 2012) presenta el desarrollo de una aplicación para el sistema operativo iOS, mediante el cual los niños con IC puedan mejorar aspectos como percepción del tono y entonación. Cabe señalar, que en México no todos los niños tienen acceso a un IC por los altos costos que representa.

En el trabajo de (Pérez-Arévalo, Manresa-Yee, y Beltrán, 2017) se propone el diseño de una aplicación móvil que permita a los niños sordos entrenar el ritmo y la coordinación a través de la generación visual y aural de ritmos. Como tal no se presentan resultados sobre el uso de la aplicación.

Por otro lado, Duarte y Tavares plantean en su artículo "A tool for the musical education of Deaf people” el desarrollo de diferentes interfaces que permitan a las personas con discapacidad 
auditiva recibir información sobre aspectos musicales. Una de las interfaces propuestas se describe como un apoyo que permite extraer elementos de la música y representarlos como elementos visuales y vibraciones a través de una aplicación (Duarte y Tavares, 2017). Con mas detalle Duarte presenta en su disertación de maestría el proceso asociado a la creación de las interfaces, destacando como principales problemas la diferenciación de vibraciones debido a la tecnología (Duarte, 2017).

Finalmente, Nakada, Onishi y Sakajiri señalan que el éxito de un artefacto o herramienta diseñada para las personas con discapacidad auditiva depende mucho de la interacción que se pueda llevar a cabo. Es por ello, que estos autores plantean un sistema interactivo que presenta la escala musical a través de actuadores hápticos que proveen los mandos vibratorios de la consola de videojuegos Nintendo Switch para el entrenamiento musical de personas con discapacidad auditiva (Nakada, Onishi, y Sakajiri, 2018).

\section{Problemática sobre las personas con discapacidad auditiva y la música}

La enseñanza y aprendizaje de la música requiere que una persona comprenda los conceptos, las técnicas, o la metodología para producir sonidos armónicos con algún instrumento. El acceso a la música en el caso de las personas oyentes resulta una actividad trivial, debido a que la audición es el elemento que les permite interactuar de manera efectiva. No obstante, para las personas con un déficit funcional que afecte al oído, el acceso a la música pudiera resultar imposible, tal y como se expuso anteriormente, sin las adecuaciones que le permitan comprender conceptos fundamentales como pueden ser la melodía, la armonía, o el ritmo.

Por otro lado, y desde la perspectiva empírica de los autores, así como también se describe en la literatura (Patterson, 2003), se constató que los profesores de música, así como el resto en general no poseen las habilidades lingüísticas adecuadas que les permita interactuar de manera efectiva con aquellas personas con déficit auditivo, en particular con aquellas personas que son usuarias de la lengua de señas.

Nogueira, Nagathil y Martin en su estudio enfocado al desarrollo de la música más accesible para las personas con sordera, mencionan que debido a que la música es un medio universal de interacción sociocultural y además desencadenador de emociones positivas, las personas con acceso limitado a la música como la comunidad sorda, enfrentan una degradación considerable en su calidad de vida, su formación personal, su creatividad, su temple, entre otras (Nogueira, Nagathil, y Martin, 2019). Así, pese a que algunos miembros de esta comunidad no se encuentran interesados en tener acceso a la música, muchos sí quieren ser incluidos como una cuestión de equidad y participación (Baijal, Kim, Branje, Russo, y Fels, 2012).

\subsection{Justificación}

La música es una expresión artística que resulta placentera para el ser humano, en virtud de ello, así como en el caso de los oyentes, las personas que viven con sordera deberían tener la oportunidad de sentir y apreciar la música sin importar la discapacidad auditiva. De acuerdo con la profesora de música Guylaine Vaillancourt, a través de esta bella arte se puede lograr encontrar un medio de expresión y de comunicación ideal en la vida del niño, favoreciendo su condición física, 
intelectual, afectiva y social (Vaillancourt, 2009). Asimismo, diversos estudios presentados en la sección de antecedentes describen la importancia de la música para el desarrollo del lenguaje.

Por ello, en este trabajo se plantea que al hacer uso de una aplicación móvil que proporcione retroalimentación con vibraciones, en conjunto con la enseñanza musical mediada con el uso de la lengua de señas, los niños sordos podrán ser introducidos a un contexto musical. De tal forma, considerando las necesidades de acceso a la música, se especula que el uso efectivo de la vibración del teléfono móvil podrá resultar significativo para transmitir los principales conceptos de la música, que a lo largo de su interacción en un curso pueda favorecer a que el niño sordo logre la comprensión de los elementos de la música.

\section{Marco teórico}

Previamente se planteó la relación estrecha entre la música y el sonido a través de las vibraciones. Particularmente se habló de una unidad de medida denominada hercio o hertz cuya representación simbólica es Hz. Dicha medida representa el número de oscilaciones, ciclos o vibraciones por segundo de un sonido. Cuanto mayor sea esta magnitud, refiriéndonos a los hercios, más agudo será el sonido resultante. En otros términos, esta medida refiere al concepto de la altura o también llamada tono (Tomasini, 2007).

Para medir el valor absoluto de los tonos se usa como referencia los $440 \mathrm{~Hz}$, utilizándose para lograr la afinación de la nota "la", siendo la frecuencia que tiene un diapasón para afinar diversos instrumentos musicales. La escala de tonos que se usa hoy en día se formalizó a partir del año 1630, gracias al matemático francés Mersenne quién formuló con precisión las reglas para afinar en su obra "Armonía Universal". En el Cuadro 1 se muestran las frecuencias aproximadas asociadas a cada nota de la escala musical.

Cuadro 1: Comparativa de las diferentes notaciones musicales y su respectiva frecuencia en her
\begin{tabular}{|c|c|c|}
\hline Frecuencia (Hz) & Nota musical & Notación musical anglosajón \\
\hline 261 & $\mathrm{Do}$ & $\mathrm{C}$ \\
\hline 293 & $\mathrm{Re}$ & $\mathrm{D}$ \\
\hline 328.8 & $\mathrm{Mi}$ & $\mathrm{E}$ \\
\hline 348.3 & $\mathrm{Fa}$ & $\mathrm{F}$ \\
\hline 391.1 & $\mathrm{Sol}$ & $\mathrm{G}$ \\
\hline 440 & $\mathrm{La}$ & $\mathrm{A}$ \\
\hline 492.7 & $\mathrm{Si}$ & $\mathrm{B}$ \\
\hline
\end{tabular}

La escala musical cuenta con siete notas musicales: do, re, mi, fa, sol, la y si. Cada una de las notas mencionadas anteriormente posee su propia frecuencia en hercios que las identifica y diferencia de las demás. Al conjunto de las siete notas en sucesión, de la nota do hasta la nota si, se le nombra octava. Cuando la escala termina, es decir si se llega a la nota si, la siguiente nota es do (Tomasini, 2007). 


\subsection{Color y música}

Al hablar de fenómenos físicos, se puede pensar en si hay alguna relación entre música y los colores. La investigación de Fuster y Pérez expone que en efecto existe una relación entre diferentes tonos de color y las notas de escala musical. Los autores argumentan que se sabe que desde hace más de trescientos años diversos profesionistas entre los que se encuentran matemáticos, físicos, músicos y pintores han propuesto su propia relación teórica entre notas musicales y colores. Las relaciones propuestas entre color y música se enfocan en comparar una sucesión de notas musicales con una sucesión de tonalidades de color. Los componentes tanto del color como del sonido son los siguientes: el color posee un tono, luminosidad y saturación; las dimensiones físicas del sonido comprenden el timbre, intensidad, duración y altura (Fuster y Pérez, 2010).

Debido a estas relaciones, diversos autores han hecho sugerencias sobre la posibilidad de combinar la música y el color considerando como variables la altura del sonido y el tono de color. Como ya se explicó, la altura es la cualidad que diferencia un sonido agudo de un sonido grave, mientras que el tono de color es el grado en el cual un estímulo es descrito como similar o diferente entre los estímulos rojo, amarillo y azul. Uno de los científicos más icónicos en adentrarse a esta cuestión fue el físico y matemático Isaac Newton, quien fue el primer investigador en lograr relacionar los colores luminosos de un prisma con las siete notas de la escala musical. En el Cuadro 2 se presenta la relación de colores y notas musicales propuesta por Newton citado por los autores anteriormente mencionados.

Cuadro 2: Relación de colores y notas musicales propuesta por Isaac Newton.

\begin{tabular}{|c|c|c|}
\hline Color & Nota musical & Notación musical anglosajón \\
\hline Rojo & $\mathrm{Re}$ & $\mathrm{D}$ \\
\hline Naranja & $\mathrm{Mi}$ & $\mathrm{E}$ \\
\hline Amarillo & $\mathrm{Fa}$ & $\mathrm{F}$ \\
\hline Verde & $\mathrm{Sol}$ & $\mathrm{G}$ \\
\hline Azul & $\mathrm{La}$ & $\mathrm{A}$ \\
\hline Índigo & $\mathrm{Si}$ & $\mathrm{B}$ \\
\hline Violeta & $\mathrm{Do}$ & $\mathrm{C}$ \\
\hline
\end{tabular}

Con respecto al enfoque de este trabajo de investigación, la asignación de colores a las notas musicales se empleó como un recurso gráfico muy importante para que el niño con discapacidad auditiva sea capaz de diferenciar cada nota. La combinación adecuada entre los tonos de color y las notas musicales podrá utilizarse de manera conjunta con las vibraciones, para que así la experiencia de usuario que experimente los niños sea más apacible.

\subsection{Métodos de transmisión musical en personas con discapacidad}

De acuerdo con Kawanobe, Kameda y Miyahara, entre los métodos para transmitir música a personas que viven con alguna discapacidad auditiva se destaca el uso de las diferentes propiedades que provee la vibración. También mencionan que los métodos de transmisión musical que se basan en vibración deben ser acompañados de otros recursos para así lograr transmitir los efectos evocados de la música. Debido a lo anterior, puede ser válido utilizar apoyos visuales para que los usuarios puedan recibir una retroalimentación adecuada sobre lo que se está transmitiendo a través de las vibraciones. Por lo tanto, mientras que la vibración es capaz de transmitir el ritmo, con 
el uso de elementos visuales se puede llegar a transmitir armonía y melodía (Kawanobe, Kameda, y Miyahara, 2003).

\section{Metodología}

A continuación, se presenta la metodología que se llevó a cabo a lo largo de este trabajo de investigación, de las cuales se destaca seis etapas importantes:

- Investigación y documentación: Contempla la revisión y consulta de diferentes fuentes de información relacionados al presente trabajo de investigación.

- Diseño de propuesta de solución: A partir de las bases teóricas recuperadas, así como la documentación de diversas fuentes de información, se procedió a llevar a cabo el diseño de la propuesta de solución. En esta etapa se realiza un análisis de requerimientos y un prototipo, tomando en cuenta patrones de diseño de interfaces y comentarios de especialistas en educación especial, así como de usuarios con sordera.

- Desarrollo de la aplicación: Conforme al diseño expresado en la fase anterior se procedió a realizar el desarrollo de la propuesta de solución, considerando los requerimientos de los usuarios y patrones de diseño.

- Pruebas: Una vez terminado el primer diseño de la propuesta de solución se procedió a realizar las pruebas correspondientes del software desarrollado con los usuarios finales. Se destaca que durante esta fase se destinó un espacio específico para llevar a cabo las pruebas, el acercamiento con especialistas en educación especial y la constante toma de notas a través de una bitácora.

- Análisis de resultados y conclusiones: En esta última fase se analizan los resultados obtenidos a partir de las respuestas de los usuarios finales, esto con el fin de retroalimentar el trabajo realizado y para plantear propuestas de mejora. De igual forma, los datos obtenidos por medio de las pruebas son analizados con el fin de realizar conclusiones si el trabajo realizado cumple con sus objetivos planteados.

\section{Diseño de la aplicación}

\subsection{Modelo basado en prototipos}

De acuerdo con Berzins, el modelo basado en prototipos es un enfoque para el desarrollo de software que hace uso de prototipos para ayudar tanto a los desarrolladores como a los usuarios finales a visualizar el sistema propuesto y predecir sus propiedades en un proceso iterativo. El prototipo de software resultante refleja con precisión los aspectos elegidos del sistema, los formatos de visualización, los valores calculados, así como los tiempos de respuesta (Berzins, 2003). No se entrará a detalle sobre la metodología basada en prototipos, sin embargo, en caso de que el lector desee conocer más al respecto se anexa la fuente bibliográfica en el apartado referencias. 
El proceso seguido consistió en la generación de un prototipo funcional a partir de los requerimientos de los usuarios finales (siendo en específico los usuarios con discapacidad auditiva). Posteriormente se llevó a cabo una demostración del resultado y validación con los expertos en educación especial tomando en cuenta su retroalimentación. Debido a que este enfoque presenta la característica de ser iterativo, se llevó a cabo un refinamiento y optimización del prototipo conforme a los comentarios de los expertos hasta satisfacer sus necesidades. Finalmente, a partir de las correspondientes implementaciones y la aprobación de los mismos se obtuvo el prototipo final de la aplicación.

\subsection{Diseño de aplicaciones para personas con discapacidad}

Los usuarios con discapacidad auditiva pueden afrontar adversidades cuando interactúan con interfaces que no contemplen en su diseño el uso de su lengua natural para la transmisión de información. Por ello es importante considerar este hecho de forma que los usuarios con discapacidad auditiva sean capaces de apropiarse de los contenidos. En este sentido, los trabajos revisados no planteaban el uso de la lengua de signos como un mecanismo de mediación con la interfaz. Por ello, con esta propuesta se aumentan la independencia, la confianza, la motivación y el contacto social de los niños que viven con una discapacidad auditiva (Al-Osaimi, AlFedaghi, y Alsumait, 2009). Por lo tanto, a continuación, se plantean ciertas recomendaciones a considerar mencionados por los autores anteriormente citados en el diseño de aplicaciones móviles para usuarios con sordera:

- Texto: La cantidad de texto debe ser de cuatro palabras por línea, proporcionando de igual manera una imagen y/o un vídeo en lengua de señas para cada concepto, definición o instrucción.

- Íconos: Usar íconos con su respectiva representación en lengua de señas en lugar de solo alguna imagen.

- Ayuda: Es importante brindar a los usuarios un apartado de ayuda a través de un vídeo en lengua de señas mostrando el cómo interactuar con la interfaz.

- Actores para los vídeos en lengua de señas: Es recomendable usar actores reales para los vídeos en Lengua de Señas.

- Sonidos: Debido a que existen diferentes niveles de pérdida auditiva en niños que viven con una discapacidad auditiva, es necesario usar sonidos claros y adecuados para mejorar su experiencia con la interfaz.

- Diseño de la interfaz: Así como los niños oyentes, los niños que padecen sordera pierden el interés por los programas educativos de manera rápida. Es por ello que es necesario diseñar interfaces coloridas y animadas para captar la atención de los usuarios.

\subsection{El uso de osciladores vibratorios en dispositivos móviles}

Una de las funcionalidades más habituales que ofrecen los dispositivos móviles es la vibración, siendo un aspecto tan común que hasta pudiera pasar desapercibido. Conforme a Egby, los 
osciladores vibratorios son una buena alternativa a fin de que las personas que tienen problemas para oír puedan mantenerse atentos o alertas a lo que sucede a su alrededor (Egby, 2020). El autor señala que hay dos formas de provocar una vibración fuerte, dependiendo de igual forma de la arquitectura del dispositivo móvil:

- Rotación con masa descentrada: Es el mecanismo más habitual para hacer vibrar a los dispositivos móviles, y consiste en un motor giratorio de corriente continua en miniatura que posee un contrapeso en el eje para desequilibrarlo.

- Aplicación de resonancia: Otra forma de lograr vibraciones en los dispositivos móviles es a través de un actuador resonante lineal, que consiste en un mecanismo del tamaño de una moneda que vibra de arriba hacia abajo.

El presente desarrollo propuesto en este artículo presenta la cualidad de hacer uso de vibraciones para proveer retroalimentación a las notas de la escala musical (tomando en cuenta las frecuencias del Cuadro 1). Sin embargo, aun cuando las tecnologías que ofrece la plataforma Android son limitadas para controlar de manera precisa el flujo de vibraciones, se logró adaptar la intensidad de cada una de las notas musicales en la aplicación.

\subsection{Arquitectura de la aplicación}

El propósito de la arquitectura de software es el de separar las preocupaciones de cada componente que la integra, logrando así un bajo acoplamiento de los componentes y la posibilidad de escalamiento. Por ello, en la Figura 1 se visualiza la arquitectura de la aplicación desarrollada, la cual consiste en una arquitectura de tres capas: capa de presentación, de servicios y de hardware. En la capa de presentación se halla la interfaz gráfica donde el usuario interactúa para manejar la aplicación. Después se muestra la capa de servicios, donde se presentan las principales funcionalidades de la herramienta, las cuales consisten en actividades para practicar notas musicales, practicar ritmo y finalmente la sección para tocar el piano. Por último, en la capa de hardware se enfatiza el uso de diferentes sensores que provee el dispositivo móvil, los cuales son los osciladores para generar vibraciones de las notas y de igual manera los altavoces que liberan los sonidos de la escala musical. 


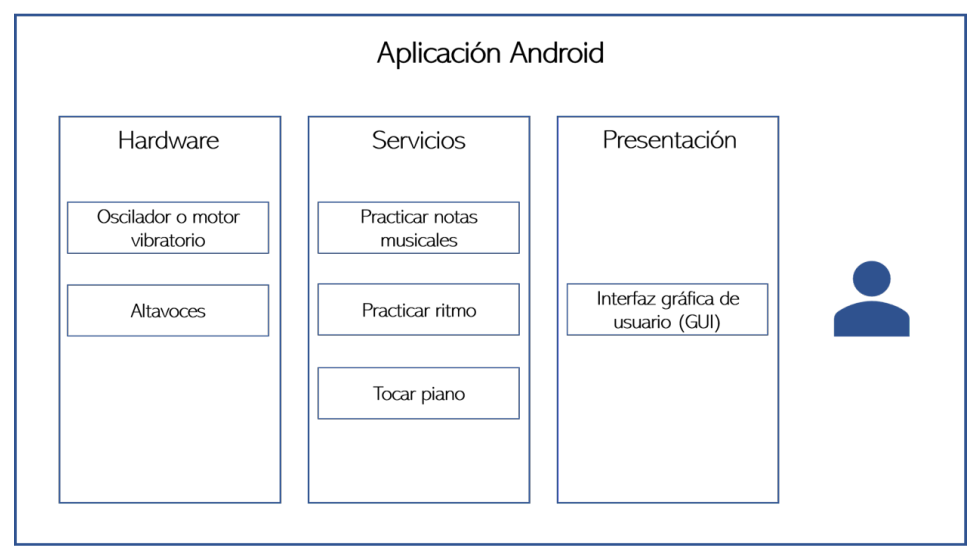

Figura 1: Arquitectura de la aplicación. Fuente: elaboración propia.

\subsection{Diagrama general de casos de uso}

Otro aspecto importante para la descripción de la aplicación consiste en la definición de sus funcionalidades. Para ello se plantea un diagrama general de casos de uso, donde se visualiza el comportamiento disponible en la aplicación para los usuarios finales (personas con discapacidad auditiva). Así, en la Figura 2 se puede apreciar que la aplicación consiste en tres funcionalidades principales: a) aprende las notas, b) descubre el ritmo y c) piano. En particular la funcionalidad de practicar notas musicales y practicar ritmo ofrecen un conjunto de niveles de dificultad para que el niño vaya practicando los conceptos asociados a la música. Para un mayor detalle sobre esta funcionalidad se recomienda leer las secciones 6.6 Prototipo de la aplicación.

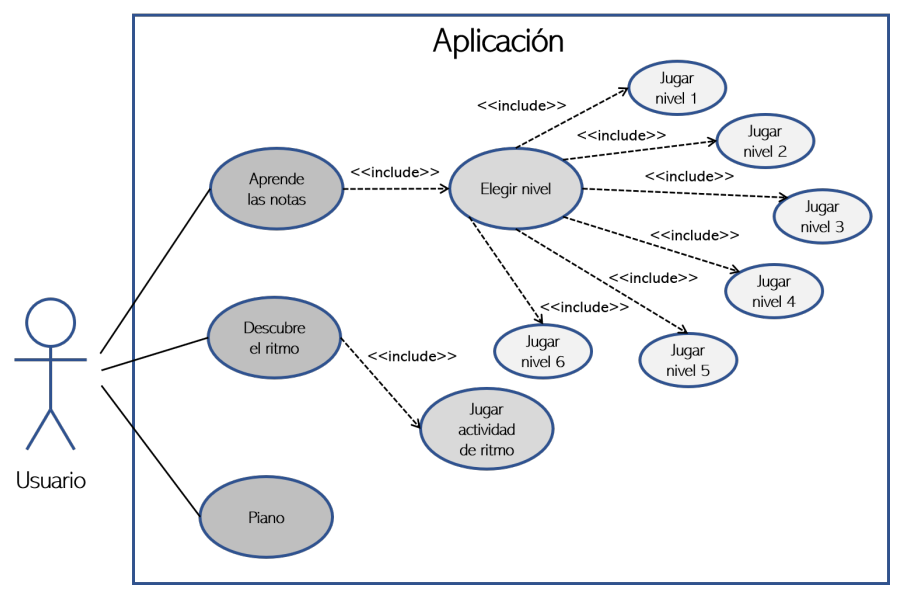

Figura 2: Arquitectura de la aplicación. Fuente: elaboración propia.

\subsection{Prototipo de la aplicación}

En esta sección se muestran algunas pantallas, así como también se describe de forma general el prototipo desarrollado. Para iniciar se presenta la pantalla splash, la cual muestra el logo de la aplicación mientras se cargan los contenidos. Consecutivamente, se presenta un vídeo de bienvenida en lengua de señas mexicana (LSM), el cual explica al usuario el contexto de la apli- 
cación y los contenidos que se abarcarán. Al cerrar el video estarán disponibles las tres funciones principales de la aplicación, véase a continuación Figura 3.
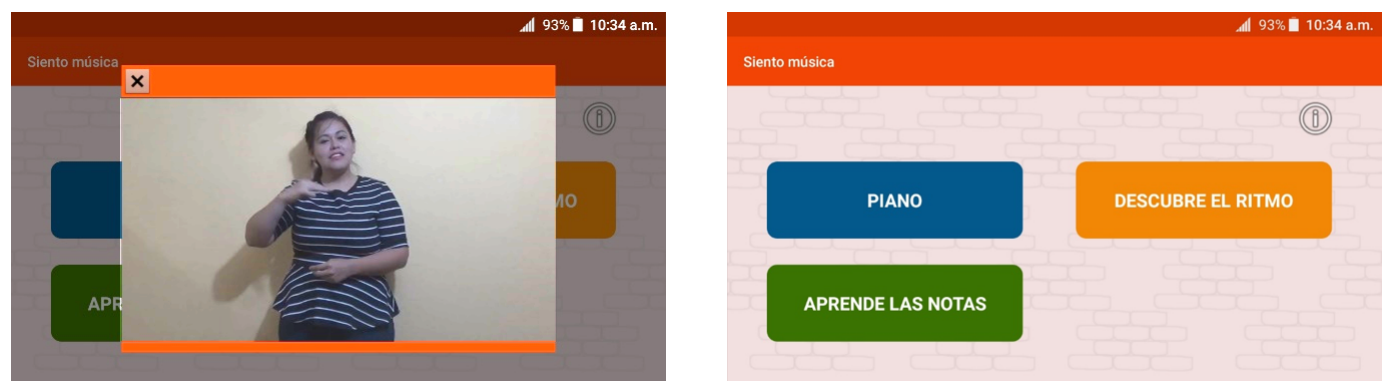

Figura 3: Prototipos de alta fidelidad: Bienvenida y menú principal. Fuente: elaboración propia.

Cabe señalar que cada apartado de la aplicación posee un vídeo de instrucciones en LSM, esto con el fin de que el niño pueda acceder fácilmente a los contenidos de lo que trata cada ejercicio de la herramienta.

\subsubsection{El piano}

Previamente se habló de la relación entre la música y los colores, por ello siguiendo los principios planteados por Isaac Newton, cada nota musical se asoció con un color tal y como lo describió el matemático en el Cuadro 2. Cuando el usuario presiona cada una de las teclas presentadas en la Figura 4, la aplicación libera la correspondiente vibración y sonido

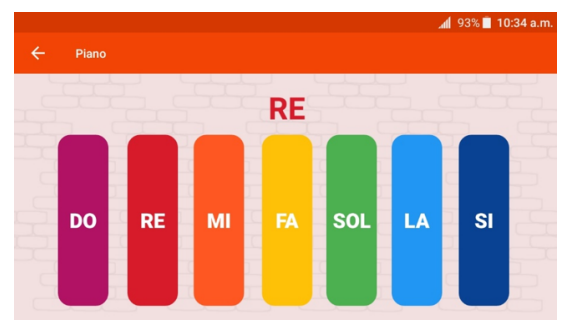

Figura 4: Prototipos de alta fidelidad: Sección Piano. Fuente: elaboración propia.

\subsubsection{Aprende las notas}

Antes de que el niño inicie con las actividades de aprendizaje de notas, la aplicación presenta un vídeo como introducción para el nivel 1. Para ello la intérprete señala que en este nivel se presentan dos notas, las cuales al tocarse liberarán una vibración. Como acción, se pide al usuario que trate de sentir la diferencia entre ellas. En la Figura 5 se presenta un mapa de navegación de actividad de reconocimiento de notas musicales correspondiente al nivel 1.

Una vez terminado el vídeo se exhiben dos botones con sus respectivos colores de acuerdo con la nota musical. La interfaz coloca una leyenda en la parte superior especificando las notas musicales que se practicarán dentro del nivel. Una vez que el usuario haya sentido las vibraciones de las notas puede proseguir tocando el botón continuar. 
Posteriormente, la aplicación pide al usuario pulsar el botón gris con una interrogación, el cual liberará una vibración, para ello el usuario deberá seleccionar de las notas presentadas en pantalla a cuál corresponde la vibración sentida.

El nivel 1 de ejercicios de reconocimiento de notas musicales fue titulado con el nombre "Conejo salta". En él se le pide al usuario, a través de un vídeo, que ayude al conejo a llegar a la zanahoria. La aplicación acompaña esta pantalla con una amigable animación de un conejo saltando. Así, mientras el usuario vaya acertando a las notas musicales el conejo se acercará más a la zanahoria.

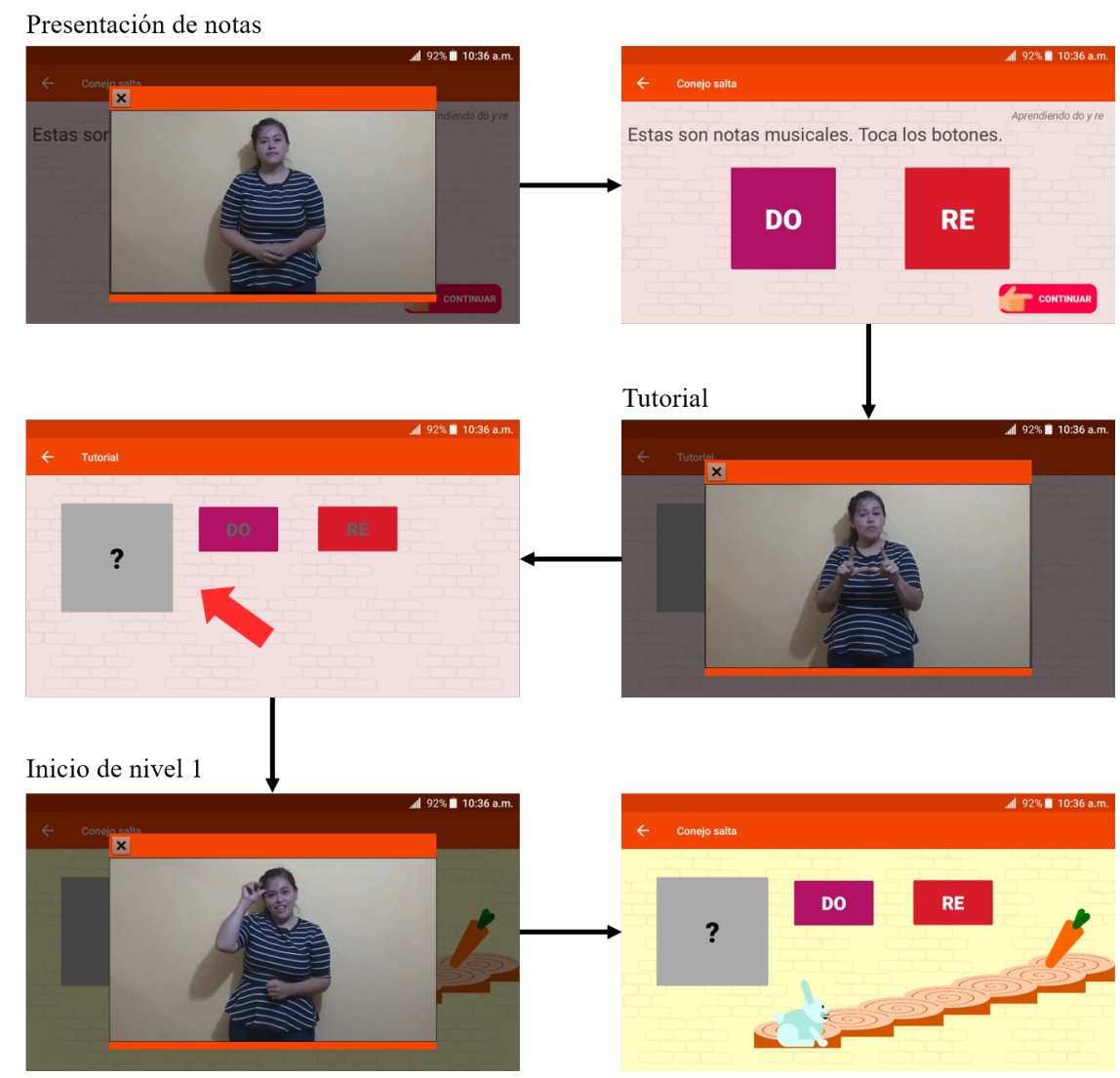

Figura 5: Prototipos de alta fidelidad: Mapa de navegación de Actividad de reconocimiento de notas musicales. Fuente: elaboración propia.

De esta forma, cada inicio de nivel posee la presentación de las notas musicales que se practicarán dentro de la actividad. Mientras el usuario vaya completando los niveles, la aplicación colocará más notas musicales con su respectiva notación de color y distintiva vibración, siendo específicos, se agrega una nota más por nivel. Cada nivel posee un vídeo explicativo, donde dependiendo del nivel se presenta un contexto diferente, es decir, la variedad de situaciones cambia. Por ejemplo, para el nivel 2 se le explica al usuario que ayude al sapo a llegar a la meta, y para el nivel 6 se le explica usuario que ayude al canguro a llegar a su casa. En la Figura 6 se presenta las imágenes demostrativas de algunos de los personajes de la aplicación. 


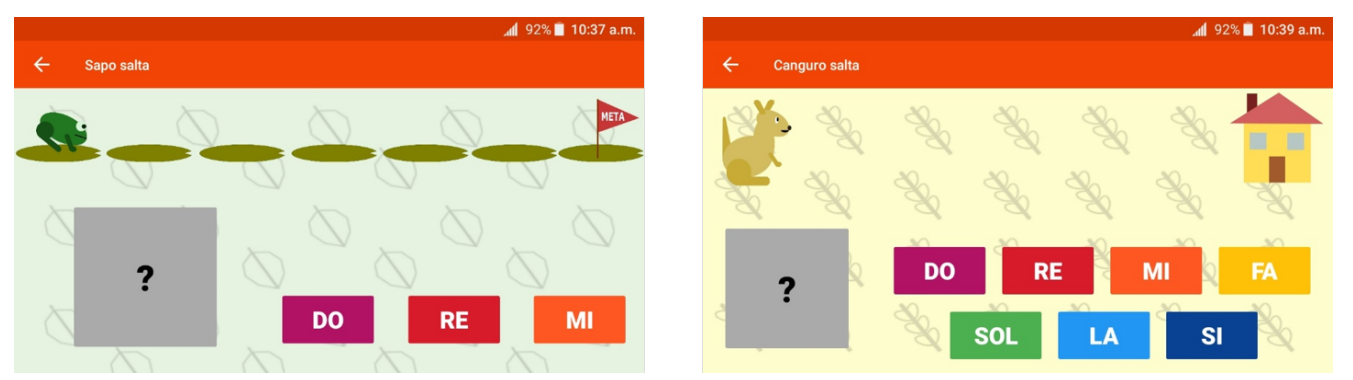

Figura 6: Prototipos de alta fidelidad: Personajes de la aplicación. Fuente: elaboración propia.

\subsubsection{Descubre el ritmo}

Para el apartado de las actividades de ritmo se tiene un vídeo donde se explica que las pelotas se mueven al ritmo de la vibración, por lo cual se le pide al usuario que seleccione la pelota que se mueva acorde al patrón rítmico. En la interfaz para las actividades de ritmo las pelotas se mueven de manera aleatoria, mientras que el dispositivo libera vibraciones cada cierto tiempo. El usuario debe seleccionar la pelota que se mueva al ritmo de la vibración, esto a través de los botones que se encuentran en la parte inferior. Las pantallas de las actividades de ritmo pueden ser visualizados en la Figura 7.
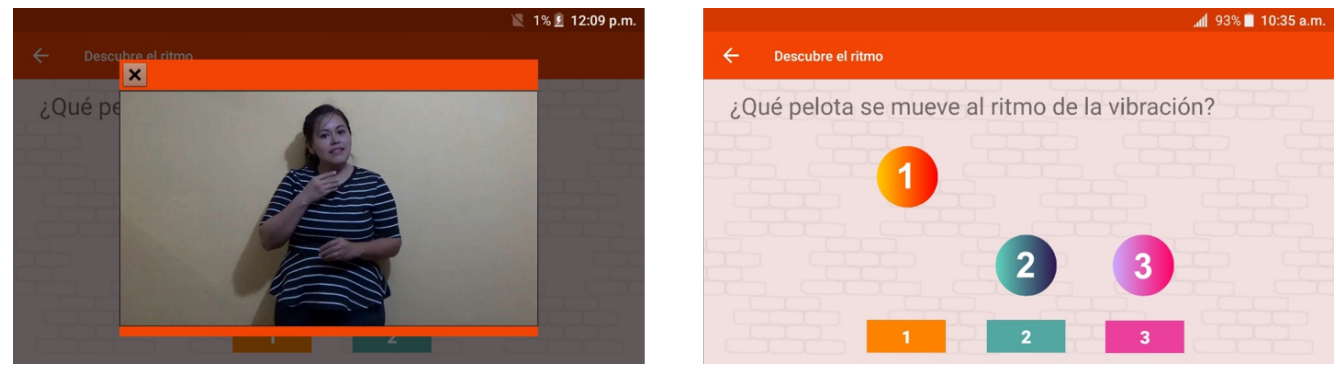

Figura 7: Prototipos de alta fidelidad: Actividades de ritmo. Fuente: elaboración propia.

\subsubsection{Retroalimentación de los ejercicios}

Cuando el usuario contesta de manera correcta en cualquiera de las actividades, la aplicación muestra un mensaje en color verde con al texto “¡Muy bien!”. Mientras que cuando el usuario realiza una respuesta errónea, la aplicación muestra un mensaje de color rojo con el texto "Intenta de nuevo" alentando a que el usuario vuelva a realizar el ejercicio. En la Figura 8 se observan los mensajes tanto de respuesta correcta como de respuesta incorrecta.

Finalmente, cabe señalar que, para la construcción de la propuesta de solución del presente trabajo de investigación, se tomaron en cuenta diversas opiniones, entre las que se encuentran un experto en educación especial con experiencia en terapia del lenguaje, maestros de educación especial usuarios de la LSM, así como consideraciones de los mismos usuarios con discapacidad auditiva. 

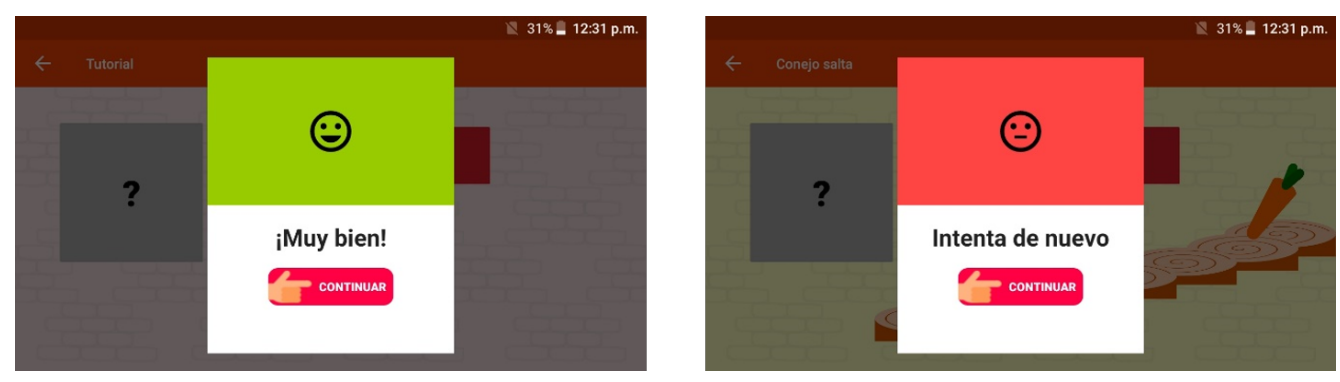

Figura 8: Prototipos de alta fidelidad: Mensajes de respuesta. Fuente: elaboración propia.

\section{Pruebas y experimentación}

Gracias al apoyo de la asociación Difusión, Inclusión y Educación del Sordo (DIES) A.C. de la ciudad de Xalapa, Veracruz, México se logró interactuar de forma inicial un grupo de niños con discapacidad auditiva. Como se mencionó al inicio del documento, el grupo contaba con una población de 12 niños con edades entre 5 y 12 años, cuyo perfil auditivo se establece como niños y niñas con sordera profunda en su mayoría.

Cabe destacar que estos niños no forman parte de un curso organizado por el estado mexicano, sino más bien se conforman con niños que requieren aprender a través de su lengua natural la LSM, y que la asociación DIES conduce de forma altruista.

De esta forma se inició el contacto con los niños y niñas a través de sesiones presenciales sabatinas en donde mediante diversas estrategias se les dio a conocer diversos conceptos de música. El planteamiento original era llevar la aplicación móvil a un salón de clases y en presencia de un profesor intérprete darle seguimiento al avance. Sin embargo, como se dijo, la pandemia provocada por COVID-19 requirió suspender toda actividad presencial, motivo por el cual, del grupo de niños originalmente trabajado, solo se pudo dar seguimiento remoto a 5 niños.

\subsection{Descripción de las sesiones presenciales}

La visita inicial a la asociación consistió en la observación de una clase de los niños con discapacidad auditiva. Las clases impartidas eran en Lengua de Señas Mexicana (LSM), y esto llevándose a cabo mediante la participación de una profesora sorda y una profesora oyente. Una vez en contexto, fue necesario realizar un análisis acerca del cómo se presentaría la aplicación a los niños. Dado que presentar la aplicación de manera apresurada pudiera causar confusión con los infantes, de acuerdo con los comentarios de sus profesoras, fue necesario realizar actividades de apoyo para que los niños entraran en contexto sobre lo que trataría la aplicación. De acuerdo con comentarios de las profesoras, la música es un concepto ambiguo para los niños debido a que la mayoría de ellos saben que existe algo llamado música y hasta ahí queda su conocimiento sobre ella.

A partir de la visita inicial a las instalaciones de la asociación DIES A.C., tomando en cuenta lo observado en la clase y lo discutido con las maestras y la especialista, se llevó a cabo una planeación educativa con el fin de establecer las actividades que se llevarían a cabo en próximas clases. Como resultado, se decidió emplear inicialmente un material didáctico el cual consistiría 
de: cuento infantil sobre música, diapositivas de introducción a la música, juego de lotería sobre música e instrumentos y notas musicales realizados con botellas rellenas de arroz.

\subsubsection{Primera sesión}

En la primera sesión con los niños se presentó mediante un proyector un cuento infantil que trataba sobre música, donde para su presentación las maestras apoyaron en la interpretación en LSM. El objetivo de la proyección era con el fin de que los alumnos entraran en contexto sobre lo que es la música. La interpretación del cuento en LSM es de suma importancia para que los niños comprendan lo que se llevará a cabo a lo largo de las sesiones.

Posteriormente, se presentaron unas diapositivas retomando conceptos musicales muy básicos: música, instrumento musical, músicos, notas musicales, entre otros más. Más tarde se expusieron los instrumentos musicales que existen, donde los niños reconocieron el violín, la flauta, el piano, el tambor y la guitarra. Seguidamente, entre todo el grupo se jugó el tradicional juego de lotería, solo que en esta versión se retomarían únicamente elementos musicales anteriormente expuestos en la sesión.

Para concretar la sesión de actividades, las profesoras pidieron a los alumnos que dibujaran su instrumento musical favorito. Dentro de los dibujos se destacó la tendencia de dibujar guitarras, pianos y hasta una batería.

\subsubsection{Segunda sesión}

Con ayuda de las profesoras de los niños se realizó una recapitulación de la sesión pasada sobre los diferentes conceptos musicales que se vieron en la clase. Posteriormente se llevó a cabo la actividad de las notas musicales mediante botellas con arroz, simulando las diferentes notas de la escala musical. Cada botella fue etiquetada con el nombre de la nota y el color asociado, siendo un recurso importante para que los niños pudieran señalarlos y diferenciarlos de manera más fácil. Al sacudir cada una de las botellas, la vibración del contenido de éstas resultaba diferente dependiendo de la nota o botella elegida.

Las profesoras les dieron instrucciones a los niños para que se dividieran en dos filas, esto porque se elaboraron dos juegos o series de siete botellas para que la actividad pudiera llevarse simultáneamente en dos grupos. Primero se presentó las siete notas musicales mediante las siete botellas plásticas con arroz señalando el nombre de la nota, el color de la nota, y se remarcó que debían sacudir la botella para que sientan la vibración. El objetivo era que los niños interactuaran con las botellas y lograran diferenciar la vibración emitida por los recipientes.

La dinámica inició repartiendo las primeras dos notas musicales do y re, y mientras las filas avanzaban y los niños sentían la diferenciación de las notas, las demás botellas eran pasadas en pares: mi y fa, sol y la, y finalmente, si y do. El resultado de la sesión fue satisfactorio y gratificante al observar que la mayoría de los niños lograban diferenciar la vibración pronunciada entre cada una de las notas, aunque a los niños más pequeños se les complicaba un poco debido a que querían usar las botellas como maracas. 


\subsubsection{Tercera sesión}

Para esta sesión se careció de la asistencia de varios niños del grupo, por lo que para las actividades se contó con la participación solamente de cinco niños con discapacidad auditiva. En la clase se repitió la misma dinámica de la actividad de las notas musicales mediante botellas con arroz, con la única diferencia de que los aciertos de los niños serían contabilizados y capturados. Cada alumno pasaría de forma individual a diferenciar dos notas musicales: la primera ronda consistiría en do y re, la segunda ronda en mi y fa, la tercera ronda sol y la, y, por último, la cuarta ronda de si y do.

Para la captura de los resultados se usaron las primeras tres letras del nombre de los niños, esto con el fin de preservar la identidad anónima de los mismos. De igual forma, se registró la participación de la maestra sorda dentro de la actividad. Los resultados de la actividad pueden ser visualizados en el Cuadro 3.

Cuadro 3: Resultados de diferenciación de notas musicales.

\begin{tabular}{|c|c|c|c|c|}
\hline Alumno & Ronda 1 & Ronda 2 & Ronda 3 & Ronda 4 \\
\hline EDS & Bien & Bien & Bien & Bien \\
\hline ANT & Falló & Falló & Falló & Bien \\
\hline GER & Falló & Falló & Bien & Falló \\
\hline JOS & Bien & Bien & Bien & Bien \\
\hline MAR & Bien & Bien & Bien & Bien \\
\hline Maestra sorda & Bien & Bien & Bien & Bien \\
\hline
\end{tabular}

En vista de que en la escala musical las notas van subiendo de tono, la siguiente dinámica consistía en ordenar las notas musicales de forma ascendente guiándose a través del sentido del tacto al sacudir las botellas y sentir las diferentes vibraciones. En esta ocasión cada alumno pasaba de manera individual e intentaba ordenar las botellas de acuerdo con la escala musical (do, re, mi, fa, sol, la y si), las cuales se encontraban en una mesa sin etiqueta y revueltas. Los resultados de la actividad pueden ser visualizados en el Cuadro 4.

Cuadro 4: Resultados de ordenar notas musicales.

\begin{tabular}{|c|c|}
\hline Alumno & Anotaciones \\
\hline EDS & Dos botellas mal posicionadas \\
\hline ANT & Dos botellas mal posicionadas \\
\hline GER & Tres botellas mal posicionadas \\
\hline JOS & Una botella mal posicionada \\
\hline MAR & Todas las botellas posicionadas correctamente \\
\hline Maestra sorda & Todas las botellas posicionadas correctamente \\
\hline
\end{tabular}

Conforme a las dinámicas llevadas a cabo en la sesión se pudo destacar que ciertos niños confundían la noción o idea de lo que se referían los conceptos de "más vibración" y "menos vibración", es decir, no comprendían a qué se refiere cuando algo tiene o emite más o menos. En este caso particular, cuál de las botellas vibraba más y cuál vibraba menos. Por otro lado, de acuerdo con las maestras, algunos niños intentaban guiarse más por el sonido que emitía la botella que por su vibración, debido a que ciertos alumnos no presentaban sordera profunda. Es de destacarse que para esta sesión fue necesario explicar a través de un pizarrón la noción de más y menos. De igual manera, para hacer que los niños obtuvieran mejores resultados era necesario que estos practicaran la misma dinámica de manera constante para así ver una mejoría. 


\subsection{Prueba de la aplicación}

Una vez concluidas las sesiones con el grupo de niños con discapacidad auditiva, se procedió a solicitar a los padres realizar la instalación de la aplicación en sus dispositivos móviles, esto con el fin de que en casa los infantes continuaran practicando en casa lo aprendido en la clase. Para ello se pidió apoyo a los tutores para que dieran seguimiento de sus hijos y reportaran semanalmente los avances, proporcionándoles para ello una bitácora de registro de actividades. Dicha bitácora tenía la fecha de uso de la aplicación, hora de inicio y fin, y finalmente, un apartado de observaciones.

Tales actividades serían revisadas semanalmente durante la clase sin embargo debido a la emergencia sanitaria causada por COVID-19 no fue posible volver a tener sesiones presenciales de seguimiento. Por otro lado, se observó una falta de respuesta de los padres para realizar la instalación y dar seguimiento de las actividades, por lo cual se decidió rediseñar la aplicación implementando una estrategia de seguimiento de forma remota para la revisión de pruebas. Una vez modificada la aplicación, se pidió el apoyo de las maestras para convencer a los padres de instalar la aplicación con la facilidad de que no tendrían que estar pendientes de las actividades a través de la bitácora.

\subsubsection{Método de recolección de datos}

De esta forma, la recolección de datos consistió en la grabación de las actividades de uso, tiempos y logros de los niños respecto de cada ejercicio planteado. Concretamente, se llevó a cabo de forma automática el registro de interacciones del niño sordo con la aplicación.

Para lograr el cometido anterior, se desarrolló una implementación en la plataforma Google Analytics, la cual permitió lograr el monitoreo de las sesiones de los niños, la hora de comienzo y fin, las secciones visitadas, las actividades llevadas a cabo y el número de intentos para acertar correctamente a los ejercicios de los niveles.

\subsubsection{Visualización de la recolección de datos}

Para la visualización de los resultados de los usuarios se desarrolló un sistema web para la concentración de datos del monitoreo de actividades de la aplicación. Se concentró el nombre de los usuarios registrados en la aplicación, su historial de actividades y sus respectivos resultados de intentos realizados con tiempo.

\subsubsection{Participantes}

Como se expresó, debido a diversas situaciones familiares y personales de los tutores de los niños, únicamente se pudo contar con la participación de cuatro niños durante el periodo que comprendió del mes de mayo a junio de 2020. Cabe señalar que este periodo se debió a que en México coincide con el fin del primer periodo de clases del sistema nacional. Para el siguiente periodo ya no se conformó el grupo de niños en la Asociación DIES. 


\subsection{Resultados obtenidos a partir del monitoreo}

De acuerdo con las estadísticas arrojadas a partir de la implementación del mecanismo de monitoreo a distancia, se logró puntualizar la interacción diaria de los usuarios, capturando que el tiempo promedio de uso diario de la aplicación fue de 7 minutos con 43 segundos. Las secciones de la aplicación más visitadas por los usuarios se presentan en el Cuadro 5.

Cuadro 5: Resultados de ordenar notas musicales.

\begin{tabular}{|c|c|}
\hline Sección & Número de visitas \\
\hline Piano & 7 \\
\hline Aprende las notas: Nivel 1 & 4 \\
\hline Aprende las notas: Nivel 2 & 4 \\
\hline Aprende las notas: Nivel 4 & 1 \\
\hline Aprende las notas: Nivel 6 & 1 \\
\hline Actividad de ritmo & 5 \\
\hline
\end{tabular}

\subsubsection{Actividades de reconocimiento de notas musicales}

Para la evaluación de los niveles se tomó en cuenta: el nivel completado, fecha de realización, subnivel (dado de que cada nivel posee subniveles), número de intentos para llegar a la nota musical correcta y tiempo transcurrido. El concepto de intento es lo importante a destacar dentro de los resultados, el cual se refiere a cuántos intentos realizó el usuario para acertar a la nota correcta.

Por ejemplo, suponga que para el nivel 1-1 el usuario presiona el botón gris y la aplicación libera la vibración correspondiente a la nota "do". El usuario analiza la respuesta vibratoria del botón y selecciona la nota re", por lo que la aplicación muestra en pantalla un mensaje de nota incorrecta registrando así un intento. El usuario recibe retroalimentación y cierra el mensaje, procede a tocar de nuevo el botón gris, siente la vibración y elige la nota "do", por lo que la aplicación muestra una animación del personaje avanzando una casilla del camino como progreso, registrando otro intento. En el proceso anterior, el monitoreo registra dos intentos para realizar el nivel 1-1, siguiendo así el monitoreo para el nivel 1-2.

A partir del mecanismo de monitoreo a distancia, se lograron capturar los resultados obtenidos de usuarios que realizaron los niveles 1 y 2 referente a las actividades de aprendizaje de notas musicales.

El nivel 1 de reconocimiento de notas musicales consiste en lo siguiente:

- El primer nivel presenta dos notas musicales: do y re.

- El personaje es posicionado en el primer escalón del camino.

- El usuario toca el botón gris y la aplicación libera la vibración correspondiente a una nota musical (pudiese ser la nota do o re).

- El usuario siente la vibración emitida y elige una opción entre los dos botones de notas musicales. 
- Si el usuario acierta la nota musical el personaje avanza, si no acierta, el personaje se mantiene en la misma posición y lo invita a que intente de nuevo.

El nivel 2 de reconocimiento de notas musicales consiste en lo siguiente:

- El primer nivel presenta dos notas musicales: do, re y mi.

- El personaje es posicionado en el primer escalón del camino.

- El usuario toca el botón gris y la aplicación libera la vibración correspondiente a una nota musical (pudiese ser la nota do, re o mi).

- El usuario siente la vibración emitida y elige una opción entre los dos botones de notas musicales.

- Si el usuario acierta la nota musical el personaje avanza, si no acierta, el personaje se mantiene en la misma posición y lo invita a que intente de nuevo.

Seguidamente en el Cuadro 6 se presenta las estadísticas del nivel 1 de la aplicación, presentando el número de intentos por usuario y el tiempo transcurrido para completar la actividad. El nombre de los niños quiénes usaron la app son colocados con las iniciales de su nombre para mantener oculta su identidad.

Cuadro 6: Resultados de intentos acumulados y tiempo por usuario para el nivel 1 de la activ
\begin{tabular}{|c|c|c|}
\hline Usuario & Intentos Nivel 1 & Tiempo (mm:ss) \\
\hline ANM & 10 & $00: 32$ \\
\hline LAI & 9 & $01: 12$ \\
\hline MAY & 12 & $00: 34$ \\
\hline ANT & 15 & $00: 42$ \\
\hline
\end{tabular}

Por otro lado, en la Figura 9 se presentan las diferentes variaciones de los resultados de los usuarios por cada subnivel para completar el nivel 1 de notas musicales, donde claramente se logra identificar que existe una moda de 2 intentos.

De acuerdo con las gráficas y los datos anteriormente presentados se puede detallar lo siguiente:

- El mayor número de intentos realizados para completar el nivel 1 fue de 15 intentos.

- El menor número de intentos realizados para completar el nivel 1 fue de 9 intentos.

- En cuanto al número de intentos capturados de los usuarios para completar el nivel 1 se presenta una moda de 2 intentos.

- De acuerdo con la suma del número de intentos totales de los usuarios, en promedio se necesitó de 12 intentos para completar el nivel 1.

- El mayor tiempo registrado para completar el nivel 1 fue de 1 minuto con 12 segundos. 


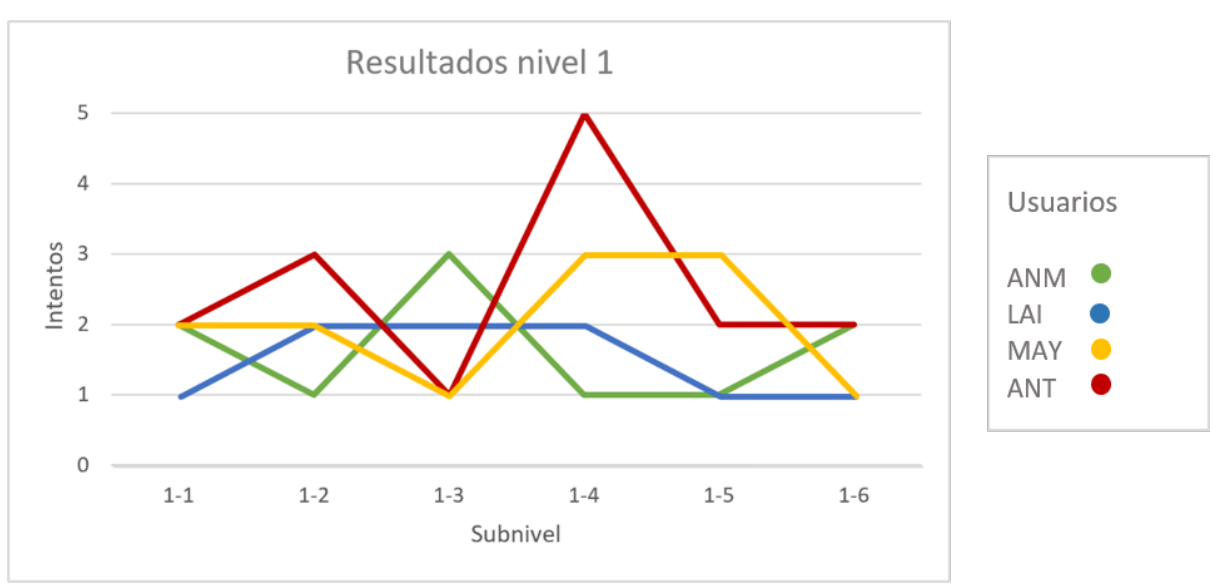

Figura 9: Resultados de nivel 1, donde se aprecia el número de intentos acumulados por cada subnivel para cada usuario. Fuente: elaboración propia.

- El menor tiempo registrado para completar el nivel 1 fue de 32 segundos.

A continuación, se presentan los resultados del usuario ANM, quien realizó el nivel 1 (notas do y re) de ejercicios de reconocimiento de notas musicales de manera completa, donde asimismo se logró capturar su progreso comparando su primera vez de uso con una segunda vez.

Conforme al Cuadro 7, el usuario registró en su primer uso de la aplicación 10 intentos para concluir el nivel 1 con un tiempo de 32 segundos.

Cuadro 7: Resultados de diferenciación de notas musicales nivel 1 (Primera vez de uso).

\begin{tabular}{|c|c|c|c|c|}
\hline Nivel & Fecha & Subnivel & Intentos & Tiempo (mm:ss) \\
\hline Nivel 1 & $27 / 05 / 2020$ & Nivel 1-1 & 2 & $00: 04$ \\
\hline Nivel 1 & $27 / 05 / 2020$ & Nivel 1-2 & 1 & $00: 05$ \\
\hline Nivel 1 & $27 / 05 / 2020$ & Nivel 1-3 & 3 & $00: 11$ \\
\hline Nivel 1 & $27 / 05 / 2020$ & Nivel 1-4 & 1 & $00: 04$ \\
\hline Nivel 1 & $27 / 05 / 2020$ & Nivel 1-5 & 1 & $00: 04$ \\
\hline Nivel 1 & $27 / 05 / 2020$ & Nivel 1-6 & 2 & $00: 04$ \\
\hline Nivel 1 & $27 / 05 / 2020$ & Total & 10 & $00: 32$ \\
\hline
\end{tabular}

De acuerdo con el Cuadro 8, el usuario registró para su segunda vez de uso 9 intentos para concluir el nivel 1 con un tiempo de 29 segundos.

Cuadro 8: Resultados de diferenciación de notas musicales nivel 1 (Segunda vez de uso).

\begin{tabular}{|c|c|c|c|c|}
\hline Nivel & Fecha & Subnivel & Intentos & Tiempo (mm:ss) \\
\hline Nivel 1 & $03 / 06 / 2020$ & Nivel 1-1 & 2 & $00: 06$ \\
\hline Nivel 1 & $03 / 06 / 2020$ & Nivel 1-2 & 2 & $00: 10$ \\
\hline Nivel 1 & $03 / 06 / 2020$ & Nivel 1-3 & 2 & $00: 05$ \\
\hline Nivel 1 & $03 / 06 / 2020$ & Nivel 1-4 & 1 & $00: 03$ \\
\hline Nivel 1 & $03 / 06 / 2020$ & Nivel 1-5 & 1 & $00: 01$ \\
\hline Nivel 1 & $03 / 06 / 2020$ & Nivel 1-6 & 1 & $00: 04$ \\
\hline Nivel 1 & $03 / 06 / 2020$ & Total & 9 & $00: 29$ \\
\hline
\end{tabular}

El progreso de avance del usuario ANM para el nivel 1 puede ser observado en la Figura 10, 
destacándose que para la segunda vez se presentó un mínimo de 1 intento y un máximo de 2 intentos, mostrándose evidentemente una mejoría.

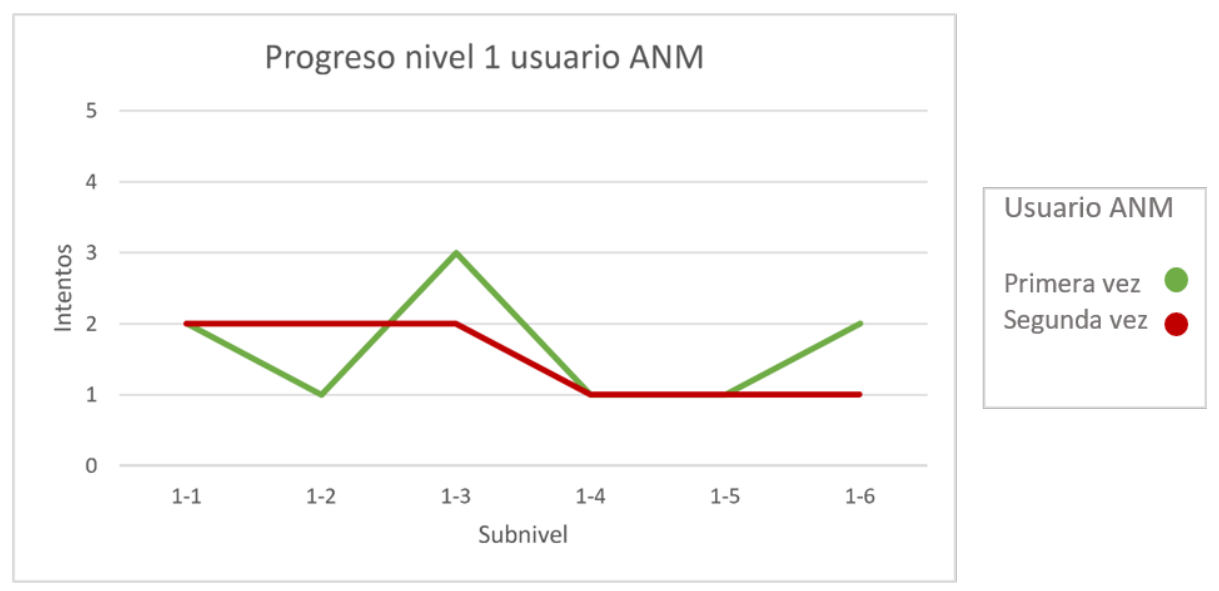

Figura 10: Progreso de ANM para el nivel 1. Fuente: elaboración propia.

A partir de los resultados recabados del usuario ANM, seguidamente se enlistan las correspondientes interpretaciones de su avance para el nivel 1:

- El usuario realizó por primera vez el nivel 1 el día 27 de mayo de 2020 y continuó la interacción hasta el día 3 de junio de 2020.

- Se identifica que cuando el usuario realizaba más intentos el tiempo era más prolongado en ciertos subniveles.

- Existió menos intentos en la segunda vez de uso, por lo consiguiente se visualiza una mejoría.

- El tiempo para realizar el nivel disminuyó entre fechas, por lo que también se considera un avance positivo.

Conforme a los datos que se presentan en el Cuadro 7 y en el Cuadro 8, se puede observar una mejoría en número de intentos y en tiempo para la realización del nivel. La primera vez que el usuario realizó el primer nivel obtuvo un total de 10 intentos para completarlo, mientras que para la segunda vez de uso completó el nivel con 9 intentos. Por otro lado, el tiempo de duración disminuyó entre las diferentes fechas, pues al principio el usuario tardó 32 segundos en terminar el nivel mientras que en la segunda vez tardó 29 segundos.

Seguidamente se presentan los resultados del usuario ANM para el nivel 2 (notas do, re y mi) de ejercicios de reconocimiento de notas musicales, analizando de igual manera su progreso entre fechas. 
Conforme al Cuadro 9, el usuario registró en su primer uso de la aplicación 12 intentos para concluir el nivel 2 con un tiempo de 38 segundos.

Cuadro 9: Resultados de diferenciación de notas musicales nivel 2 (Primera vez de uso).

\begin{tabular}{|c|c|c|c|c|}
\hline Nivel & Fecha & Subnivel & Intentos & Tiempo (mm:ss) \\
\hline Nivel 2 & $27 / 05 / 2020$ & Nivel 2-1 & 2 & $00: 03$ \\
\hline Nivel 2 & $27 / 05 / 2020$ & Nivel 2-2 & 3 & $00: 07$ \\
\hline Nivel 2 & $27 / 05 / 2020$ & Nivel 2-3 & 1 & $00: 15$ \\
\hline Nivel 2 & $27 / 05 / 2020$ & Nivel 2-4 & 1 & $00: 04$ \\
\hline Nivel 2 & $27 / 05 / 2020$ & Nivel 2-5 & 2 & $00: 08$ \\
\hline Nivel 2 & $27 / 05 / 2020$ & Nivel 2-6 & 3 & $00: 11$ \\
\hline Nivel 2 & $27 / 05 / 2020$ & Total & 12 & $00: 38$ \\
\hline
\end{tabular}

De acuerdo con la Cuadro 10, el usuario registró para su segunda vez de uso 7 intentos para concluir el nivel 2 con un tiempo de 32 segundos.

Cuadro 10: Resultados de diferenciación de notas musicales nivel 2 (Segunda vez de uso).

\begin{tabular}{|c|c|c|c|c|}
\hline Nivel & Fecha & Subnivel & Intentos & Tiempo (mm:ss) \\
\hline Nivel 2 & $03 / 06 / 2020$ & Nivel 2-1 & 1 & $00: 07$ \\
\hline Nivel 2 & $03 / 06 / 2020$ & Nivel 2-2 & 2 & $00: 07$ \\
\hline Nivel 2 & $03 / 06 / 2020$ & Nivel 2-3 & 1 & $00: 04$ \\
\hline Nivel 2 & $03 / 06 / 2020$ & Nivel 2-4 & 1 & $00: 04$ \\
\hline Nivel 2 & $03 / 06 / 2020$ & Nivel 2-5 & 1 & $00: 04$ \\
\hline Nivel 2 & $03 / 06 / 2020$ & Nivel 2-6 & 1 & $00: 06$ \\
\hline Nivel 2 & $03 / 06 / 2020$ & Total & 7 & $00: 32$ \\
\hline
\end{tabular}

El progreso de avance del usuario ANM para el nivel 2 puede ser observado en la Figura 11, destacándose que para la segunda vez se presentó un mínimo de 1 intento y un máximo de 2 intentos, mostrándose una mejoría de igual forma como lo sucedido en el nivel 1.

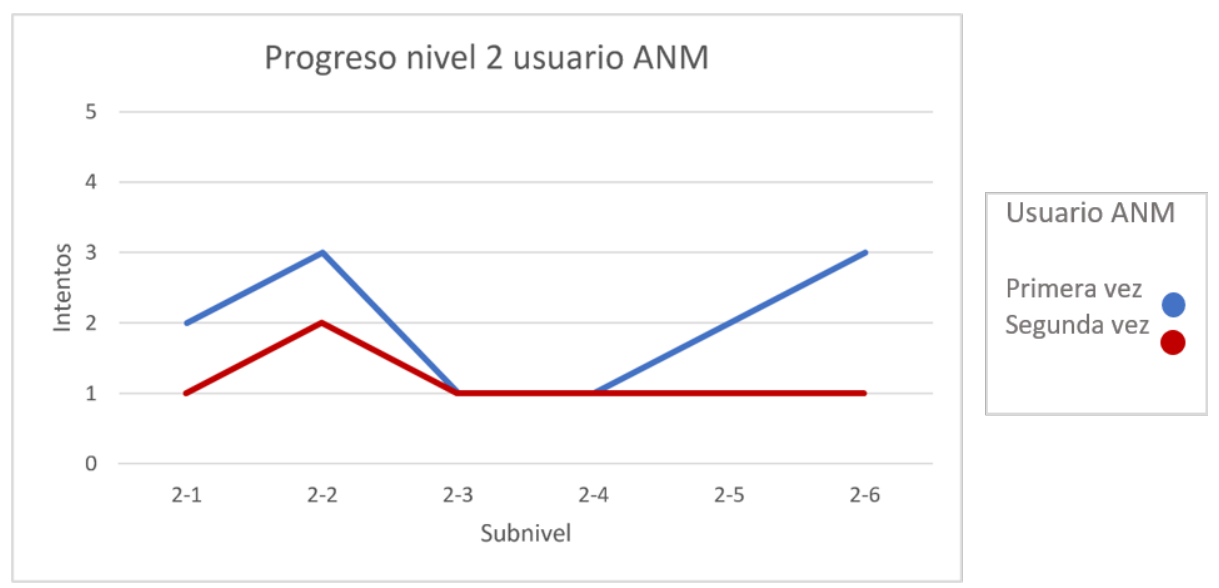

Figura 11: Progreso de ANM para el nivel 2. Fuente: elaboración propia.

A partir de los resultados recabados del usuario ANM, seguidamente se enlistan las correspondientes interpretaciones de su avance para el nivel 2: 
- El usuario realizó por primera vez el nivel el día 27 de mayo de 2020 y continuó la interacción hasta el día 3 de junio de 2020.

- Se identifica que cuando el usuario realizaba más intentos el tiempo era más prolongado en algunos subniveles.

- Existió menos intentos en la segunda vez de uso, por lo consiguiente se visualiza una mejoría.

- El tiempo para realizar el nivel disminuyó entre fechas, por lo que también se considera un avance positivo.

Así como en los resultados obtenidos en el nivel 1, en el Cuadro 9 y en el Cuadro 10 se logra visualizar una mejoría aún más ventajosa en número de intentos y en tiempo de realización del nivel. La primera vez que el usuario realizó el segundo nivel obtuvo un total de 12 intentos para completarlo, mientras que para la segunda vez de uso completó el nivel con 7 intentos. Por otro parte, el tiempo de duración disminuyó entre las diferentes fechas, pues a principio el usuario tardó 38 segundos en terminar el nivel mientras que en la segunda vez tardó 32 segundos.

Cabe destacar que mientras haya menos intentos en el transcurso de la realización de los niveles, el avance del usuario será significativo, mejorando sus habilidades para reconocer las notas de la escala musical. Con el análisis de los resultados del usuario ANM se puede esperar o especular que para los demás usuarios exista una mejoraría tanto en intentos como en tiempo para completar tanto el nivel 1 como el 2, debido a que los resultados (en este caso de ANM) arrojan cifras bastantes favorables cuando se analiza el progreso entre fechas.

\subsubsection{Actividades de ritmo}

Del mismo modo, tal como se realizó con las actividades de reconocimiento de notas musicales, se capturaron los resultados de los usuarios que llevaron a cabo las actividades de ritmo. Al igual que como las actividades de reconocimiento de notas musicales, se tomó a consideración el número de intentos para terminar la actividad y el tiempo tomado para completar el nivel.

La actividad de ritmo consiste en lo siguiente:

- En pantalla se presentan una serie de pelotas moviéndose en un patrón en específico, mientras la aplicación libera vibraciones siguiendo el ritmo del movimiento de estos.

- El usuario siente el ritmo de las vibraciones liberadas por la aplicación y selecciona la pelota que se mueve acorde al patrón vibratorio.

- Si el usuario acierta la pelota correcta pasa al siguiente subnivel, si no acierta tiene que intentar de nuevo.

A continuación, en el Cuadro 11 se presenta las estadísticas de la actividad de ritmo, presentando el número de intentos por usuario y el tiempo transcurrido para completar la actividad. 
Cuadro 11: Resultados de actividad de ritmo.

\begin{tabular}{|c|c|c|}
\hline Usuario & Intentos Nivel 1 & Tiempo (mm:ss) \\
\hline MAR & 16 & $00: 28$ \\
\hline ANT & 18 & $00: 29$ \\
\hline LAI & 16 & $01: 32$ \\
\hline ANM & 22 & $03: 01$ \\
\hline
\end{tabular}

Por otro lado, en la Figura 12 se presentan las diferentes variaciones de los resultados de los usuarios para completar la actividad de ritmo, donde claramente se logra identificar que existe una moda de 2 intentos.

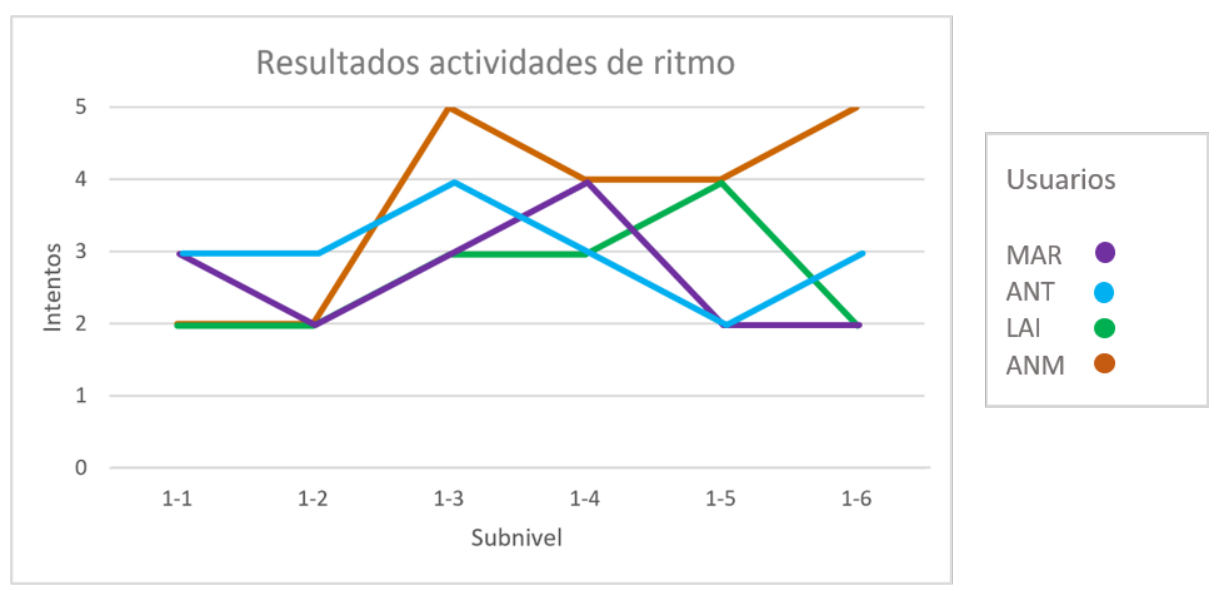

Figura 12: Resultados de actividad de ritmo, donde se aprecia el número de intentos acumulados por cada subnivel para cada usuario. Fuente: elaboración propia.

De acuerdo con las gráficas y los datos anteriormente presentados se puede detallar lo siguiente:

- El mayor número de intentos realizados para completar el nivel fue de 22 intentos.

- El menor número de intentos realizados para completar el nivel fue de 16 intentos.

- En cuanto al número de intentos capturados de los usuarios para completar el nivel se presenta una moda de 2 intentos.

- De acuerdo con la suma del número de intentos totales de los usuarios, en promedio se necesitó de 18 intentos para completar la actividad de ritmo.

- El mayor tiempo registrado para completar el nivel fue de 3 minutos con 1 segundo.

- El menor tiempo registrado para completar el nivel 1 fue de 28 segundos.

Seguidamente, se presentan los resultados del usuario ANM, quien realizó las actividades de ritmo de manera completa, donde además se logró capturar su progreso comparando su primera vez de uso con una segunda vez.

Conforme al Cuadro 12 , el usuario registró en su primer uso de la aplicación 22 intentos para concluir la actividad de ritmo con un tiempo de 3 minutos con 1 segundo. 
Cuadro 12: Resultados de actividades de ritmo (Primera vez de uso).

\begin{tabular}{|c|c|c|c|c|}
\hline Nivel & Fecha & Subnivel & Intentos & Tiempo (mm:ss) \\
\hline Actividad de ritmo & $27 / 05 / 2020$ & Ritmo 1-1 & 2 & $00: 20$ \\
\hline Actividad de ritmo & $27 / 05 / 2020$ & Ritmo 1-2 & 2 & $00: 26$ \\
\hline Actividad de ritmo & $27 / 05 / 2020$ & Ritmo 1-3 & 5 & $00: 46$ \\
\hline Actividad de ritmo & $27 / 05 / 2020$ & Ritmo 1-4 & 4 & $00: 30$ \\
\hline Actividad de ritmo & $27 / 05 / 2020$ & Ritmo 1-5 & 4 & $00: 25$ \\
\hline Actividad de ritmo & $27 / 05 / 2020$ & Ritmo 1-6 & 5 & $00: 34$ \\
\hline Actividad de ritmo & $27 / 05 / 2020$ & Total & 22 & $03: 01$ \\
\hline
\end{tabular}

De acuerdo con el Cuadro 13, el usuario registró para su segunda vez de uso 19 intentos para concluir la actividad de ritmo con un tiempo de 2 minutos con 19 segundos.

Cuadro 13: Resultados de actividades de ritmo (Segunda vez de uso).

\begin{tabular}{|c|c|c|c|c|}
\hline Nivel & Fecha & Subnivel & Intentos & Tiempo (mm:ss) \\
\hline Actividad de ritmo & $03 / 06 / 2020$ & Ritmo 1-1 & 2 & $00: 39$ \\
\hline Actividad de ritmo & $03 / 06 / 2020$ & Ritmo 1-2 & 3 & $00: 17$ \\
\hline Actividad de ritmo & $03 / 06 / 2020$ & Ritmo 1-3 & 3 & $00: 20$ \\
\hline Actividad de ritmo & $03 / 06 / 2020$ & Ritmo 1-4 & 3 & $00: 24$ \\
\hline Actividad de ritmo & $03 / 06 / 2020$ & Ritmo 1-5 & 4 & $00: 27$ \\
\hline Actividad de ritmo & $03 / 06 / 2020$ & Ritmo 1-6 & 4 & $00: 12$ \\
\hline Actividad de ritmo & $03 / 06 / 2020$ & Total & 19 & $02: 19$ \\
\hline
\end{tabular}

El progreso de avance del usuario ANM para las actividades de ritmo puede ser observado en la Figura 13, destacándose que para la segunda vez se presentó un mínimo de 2 intentos y un máximo de 4 intentos, mostrándose una mejoría.

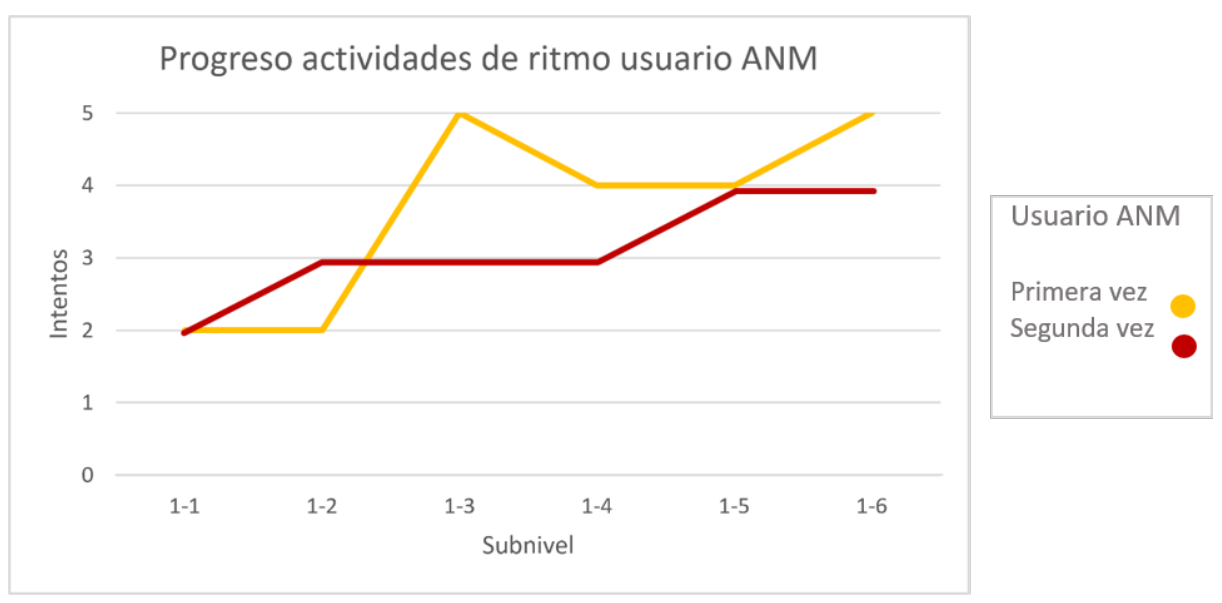

Figura 13: Progreso ANM para la actividad de ritmo. Fuente: elaboración propia.

A partir de los resultados recabados del usuario ANM, seguidamente se enlistan las correspondientes interpretaciones de su avance para la actividad de ritmo.

- El usuario realizó por primera vez las actividades de ritmo el día 27 de mayo de 2020 y continuó la interacción hasta el día 3 de junio de 2020. 
- Se identifica que cuando el usuario realizaba más intentos el tiempo era más prolongado en algunos subniveles.

- Existió menos intentos en la segunda vez de uso, por lo consiguiente se visualiza una mejoría.

- El tiempo para realizar el nivel disminuyó entre fechas, por lo que se considera un avance positivo.

Tanto en el Cuadro 12 como en el Cuadro 13 se pueden visualizar resultados positivos referente al número de intentos para concluir las actividades de ritmo. El número de intentos para completar la actividad de ritmo pasó de ser 22 intentos en la primera vez a disminuir a 19 intentos para la segunda vez, por lo cual se puede destacar una mejoría y un avance significativo en el usuario para diferenciar patrones de ritmo. Asimismo, el tiempo para concluir el nivel de ritmo redució entre las diferencias fechas, dado que al principio el usuario tardó 3 minutos con 1 segundo para terminar la actividad, mientras que en la segunda vez tardó 2 minutos con 19 segundos.

Una vez más se obtienen resultados favorables en la actividad de ritmo, lográndose reflejar un progreso bastante significativo para el usuario, quedando en evidencia sus habilidades para identificar patrones de ritmo tanto de manera sensorial como visual gracias a las vibraciones. Para esta evaluación se consideró los resultados del usuario ANM, por lo tanto, se puede estimar resultados positivos para los demás usuarios tanto en intentos como en tiempo para completar la actividad de ritmo.

\section{Conclusiones y trabajo futuro}

La asistencia a clases presenciales en la asociación DIES A.C. permitió evaluar el nivel de comprensión musical que presentaba un grupo de niños que viven con discapacidad auditiva. A partir de lo anterior, se logró observar que la mayoría de los niños demostraron interés hacia la música, tanto así que incluso tenían el conocimiento de ciertos instrumentos musicales. Por otra parte, se destacó que uno de los alumnos (siendo en específico el usuario ANM) quién posee sordera profunda asistía a clases particulares de piano.

Con base en las clases observadas se elaboró la planeación de actividades para que estas fueran impartidas presencialmente con la finalidad de que los niños entraran en contexto con los contenidos que serían presentados posteriormente como parte de la aplicación. Las sesiones permitieron que los alumnos comprendieran los principales conceptos de la música, viéndose reflejado lo aprendido en las actividades didácticas aplicadas, las cuales obtuvieron resultados positivos constatando en la expresión de los niños y en la participación de los mismos. La asistencia a clases particulares con niños con discapacidad auditiva permitió contextualizar con los usuarios finales, los cuales serían quienes usarían la aplicación móvil.

Como resultado se desarrolló una aplicación móvil que permite a niños que viven con discapacidad auditiva experimentar el significado de las notas musicales y el concepto de ritmo. Para ello se emplearon los altavoces y el oscilador o motor vibratorio, así como el uso de los colores, explotando las capacidades del teléfono para brindar una experiencia única dentro del software. 
Debido a la contingencia causada por el virus COVID-19 se consiguió alternar el seguimiento de cada niño con un mecanismo de monitoreo a distancia para evaluar y recabar resultados.

También fue posible evaluar, la aplicación final por expertos en educación especial, quiénes respondieron con aceptación acerca de los contenidos y métodos abordados en la aplicación. Tras las pruebas con la aplicación con niños con sordera se logró demostrar que el uso de vídeos de instrucciones en LSM, íconos representativos, interfaces llamativas, y por supuesto el uso de vibraciones, fueron significativas para fomentar el uso de la aplicación.

Como aspecto colateral, el tutor de uno de los niños comentó la experiencia de uso de su hijo, quién a partir de usar la aplicación se le despertó la afición por el piano, esto debido a que en la sección de "Piano" de la herramienta permite tocar de manera libre las notas musicales, recibiendo como retroalimentación vibración y sonido.

Gracias a los contenidos y actividades presentadas en la herramienta, se logró evaluar la aplicación con niños que viven con una discapacidad auditiva. Conforme a los resultados obtenidos, se identificó una mejoría en cuanto a las actividades de reconocimiento de notas musicales y los ejercicios de ritmo, esto bajo un enfoque cualitativo. El número de intentos para terminar los ejercicios y el tiempo para completarlos fueron positivos, pues las cifras son menores a comparación del primer uso de la aplicación al analizar el progreso entre fechas. Es importante mencionar que la habilidad de reconocimiento de notas musicales y la identificación de patrones de ritmo es un proceso de aprendizaje que se obtiene a través de práctica, entrenamiento y constancia, por lo que mientras el usuario practique de manera constante, los resultados serán cada vez mejores.

Dada la contingencia sanitaria, únicamente se logró hacer el pilotaje de la aplicación con cierto número de usuarios con sordera, sin embargo, se logró encontrar una mejoría en cuanto a la identificación de notas musicales y patrones de ritmo bajo un enfoque cuantitativo. No obstante, se espera realizar pruebas con un mayor rango de usuarios, y esto con el fin de observar la interacción que realizan los usuarios, así como sus reacciones al estar manipulando la aplicación.

Para concluir, uno de los comentarios recibidos por parte de especialistas en educación especial fue que la elaboración de productos, proyectos o herramientas para la comunidad sorda es bastante significativo y valioso. Es por ello por lo que es importante promover el desarrollo de nuevas tecnologías para personas que viven una discapacidad auditiva, logrando así una sociedad incluyente y justa para todos.

\subsection{Trabajo futuro}

Como trabajo futuro se propone acrecentar los contenidos de la aplicación a fin de reforzar los ejercicios de ritmo y actividades de reconocimiento musical. De igual manera, se plantea agregar actividades que sirvan como introducción musical, abarcando conceptos y elementos musicales para que así los usuarios tengan más nociones, contacto y contexto hacia a la música.

Conforme a la fecha que dicta este documento, las tecnologías que provee la plataforma Android, así como las características técnicas que poseen los dispositivos móviles del momento, son ciertamente limitadas para controlar el flujo de vibraciones. Se espera que en un futuro se pueda hacer uso de aditamentos externos o incluso la introducción de osciladores más sofisticados para controlar la intensidad de la vibración, pudiéndose especular que se logre mejorar la experiencia de uso de la aplicación de apoyo a la apreciación musical. 
Desde otra perspectiva, es interesante plantear la migración de la aplicación a una interfaz tangible por medio de un entorno físico, donde por medio de motores vibratorios se pueda manipular la vibración de las diferentes notas musicales. Asimismo, la introducción de una nueva interfaz avanzada implica contemplar la modalidad en la que se presentarían los contenidos, así como la interacción que llevaría a cabo los usuarios con el futuro sistema en cuestión. 


\section{Referencias}

Al-Osaimi, A., AlFedaghi, H., y Alsumait, A. (2009). User interface requirements for e-learning program designed for deaf children. En Proceedings of the first kuwait conference on $e$ services and e-systems. New York, NY, USA: Association for Computing Machinery. doi: 10.1145/1836029.1836036 [GS Search]

Baijal, A., Kim, J., Branje, C., Russo, F., y Fels, D. I. (2012). Composing vibrotactile music: A multi-sensory experience with the emoti-chair. En 2012 ieee haptics symposium (haptics) (p. 509-515). doi: 10.1109/HAPTIC.2012.6183839 [GS Search]

Berzins, V. (2003). Software prototyping. En Encyclopedia of computer science (p. 1636-1638).

Caicedo, L. (2015, 12). La sordera: una oportunidad para descubrir la música. Revista Española de Discapacidad, 3, 133-137. doi: 10.5569/2340-5104.03.02.09 [GS Search]

CONADIS (2016). Lengua de señas mexicana (lsm). https://www.gob.mx/conadis/articulos/ lengua-de-senas-mexicana-lsm?idiom=es. Accedido el 20-01-2021.

Cuena, C. A. (2015). ¿qué dicen los resultados de mis pruebas audiológicas? https://www.centroauditivo-valencia.es/2015/04/15/qu\%C3\%A9-dicen-los-resultados -de-mis-pruebas-audiol\%C3\%B3gicas/. Accedido el 15-05-2021.

Darrow, A. A. (1985). Music for the deaf. Music Educators Journal, 71(6), 33-35. doi: $10.2307 / 3396472$ [GS Search]

Darrow, A. A. (2007). Teaching students with hearing losses. General Music Today, 20(2), 27-30. doi: 10.1177/10483713070200020107 [GS Search]

Duarte, E. G. (2017). Uma ferramenta para a educação musical dos surdos. Tesis de Master no publicada, Universidade Estadual de Campinas, Faculdade de Engenharia Elétrica e de Computação, Campinas, SP. http://repositorio.unicamp.br/jspui/handle/REPOSIP/330995. Accedido el 20-05-2021.

Duarte, E. G., y Tavares, T. F. (2017, sep). A tool for the musical education of deaf people. En Proceedings of the 16th brazilian symposium on computer music. http://compmus.ime .usp.br/sbcm/2017/assets/SBCM2017Proceedings.pdf\#page=164. Accedido el 20-05-2021. [GS Search]

Egby, F. (2020). What makes your smartphone vibrate? https://medium.com/everyday-science/ what-makes-your-smartphone-vibrate-9f3670e74759. Accedido el 22-05-2020.

FIAPAS (2005). Qué es la sordera. http://www.fiapas.es/que-es-la-sordera. Accedido el 20-012020.

Fourney, D. W., y Fels, D. I. (2009). Creating access to music through visualization. En 2009 ieee toronto international conference science and technology for humanity (tic-sth) (p. 939-944). doi: 10.1109/TIC-STH.2009.5444364 [GS Search]

Fuster, J., y Pérez, E. (2010, 01). Color y música: relaciones físicas entre tonos de color y notas musicales.

[GS Search]

Hash, P. (2003). Teaching instrumental music to deaf and hard of hearing students. Research \& Issues in Music Education, 1(1). [GS Search]

Innes-Brown, H., Marozeau, J. P., Storey, C. M., y Blamey, P. J. (2013, octubre). Tone, rhythm, and timbre perception in school-age children using cochlear implants and hearing aids. Journal of the American Academy of Audiology, 24(09), 789-806. doi: 10.3766/jaaa.24.9.4 [GS Search] 
Karam, M., Russo, F. A., y Fels, D. I. (2009). Designing the model human cochlea: An ambient crossmodal audio-tactile display. IEEE Transactions on Haptics, 2(3), 160-169. doi: 10.1109/TOH.2009.32 [GS Search]

Kawanobe, M., Kameda, M., y Miyahara, M. (2003). Corresponding affect between music and color. En Smc'03 conference proceedings. 2003 ieee international conference on systems, man and cybernetics. conference theme - system security and assurance (cat. no.03ch37483) (Vol. 5, p. 4190-4197). doi: 10.1109/ICSMC.2003.1245643 [GS Search]

McCord, K. (2001). Music software for special needs: Music educators faced with adapting their instruction for children with special needs can find effective tools in computer software. Music Educators Journal, 87(4), 30-64. doi: 10.2307/3399722 [GS Search]

McDowell, C. (2010). An adaption tool kit for teaching music. Teaching exceptional children plus, 6(3), 20. [GS Search]

Musical vibrations. (2010). https://www.musicalvibrations.com/. Accedido el 20-01-2021.

Music and the deaf. (2019). https://www.matd.org.uk/. Accedido 20-01-2021.

Nakada, K., Onishi, J., y Sakajiri, M. (2018). An interactive musical scale presentation system via tactile sense through haptic actuator. En 2018 ieee international symposium on haptic, audio and visual environments and games (have) (p. 1-4). doi: 10.1109/HAVE.2018.8548061 [GS Search]

Nogueira, W., Nagathil, A., y Martin, R. (2019). Making music more accessible for cochlear implant listeners: Recent developments. IEEE Signal Processing Magazine, 36(1), 115127. doi: 10.1109/MSP.2018.2874059 [GS Search]

Oxford (2019). Música. https://www.lexico.com/es/definicion/musica. Accedido el 20-01-2020.

Patterson, A. (2003). Music teachers and music therapists: Helping children together. Music Educators Journal, 89(4), 35-38.

Pérez-Arévalo, C., Manresa-Yee, C., y Beltrán, V. M. P. (2017). Game to develop rhythm and coordination in children with hearing impairments. En Proceedings of the xviii international conference on human computer interaction. New York, NY, USA: Association for Computing Machinery. doi: 10.1145/3123818.3123853 [GS Search]

Tomasini, M. C. (2007, October). El fundamento matemático de la escala musical y sus raíces pitagóricas. Revista Ciencia y Tecnología: Facultad de Ingeniería de la Universidad de Palermo, 15-28. [GS Search]

Torppa, R., y Huotilainen, M. (2019). Why and how music can be used to rehabilitate and develop speech and language skills in hearing-impaired children. Hearing Research, 380, 108-122. doi: 10.1016/j.heares.2019.06.003 [GS Search]

Vaillancourt, G. (2009). Música y musicoterapia: Su importancia en el desarrollo infantil. Narcea Ediciones.

Zhou, Y., Sim, K. C., Tan, P., y Wang, Y. (2012). Mogat: Mobile games with auditory training for children with cochlear implants. En Proceedings of the 20th acm international conference on multimedia (p. 429-438). New York, NY, USA: Association for Computing Machinery. doi: 10.1145/2393347.2393409 [GS Search] 